\title{
Machine Learning or Econometrics for Credit Scoring: Let's Get the Best of Both Worlds*
}

\author{
Elena, Dumitrescu† Sullivan, Huéł Christophe, Hurlin§, Sessi, Tokpavi $₫$
}

October 18, 2020

\begin{abstract}
In the context of credit scoring, ensemble methods based on decision trees, such as the random forest method, provide better classification performance than standard logistic regression models. However, logistic regression remains the benchmark in the credit risk industry mainly because the lack of interpretability of ensemble methods is incompatible with the requirements of financial regulators. In this paper, we propose to obtain the best of both worlds by introducing a high-performance and interpretable credit scoring method called penalised logistic tree regression (PLTR), which uses information from decision trees to improve the performance of logistic regression. Formally, rules extracted from various short-depth decision trees built with pairs of predictive variables are used as predictors in a penalised logistic regression model. PLTR allows us to capture non-linear effects that can arise in credit scoring data while preserving the intrinsic interpretability of the logistic regression model. Monte Carlo simulations and empirical applications using four real credit default datasets show that PLTR predicts credit risk significantly more accurately than logistic regression and compares competitively to the random forest method.
\end{abstract}

JEL Classification: G10 C25, C53

Keywords: Risk management; Credit scoring; Machine learning; Interpretability; Econometrics.

*This paper has previously circulated under the title "Machine Learning for Credit Scoring: Improving Logistic Regression with Non-Linear Decision-Tree Effects". We are grateful to Emanuele Borgonovo (the editor), two anonymous referees, Jérémy Leymarie, Thomas Raffinot, Benjamin Peeters, Alexandre Girard, and Yannick Lucotte. We also thank the seminar participants at University of Orléans as well as the participants of the 16th Conference "Développements Récents de l'Econométrie Appliquée la Finance" (Université Paris Nanterre), 7th PhD Student Conference in International Macroeconomics and Financial Econometrics, 35th Annual Conference of the French Finance Association and International Association for Applied Econometrics for their comments. Finally, we thank the ANR programs MultiRisk (ANR-16CE26-0015-01), CaliBank (ANR-19-CE26-0002-02), and the Chair ACPR/Risk Foundation: Regulation and Systemic Risk for supporting our research.

${ }^{\dagger}$ EconomiX-CNRS, University of Paris Nanterre, 200 Avenue de la République, 92000 Nanterre, France. E-mail: elena.dumitrescu@parisnanterre.fr

${ }^{\ddagger}$ Corresponding author, Univ. Orléans, CNRS, LEO (FRE 2014), Rue de Blois, 45067 Orléans. E-mail: sullivan.hue@univ-orleans.fr

§Univ. Orléans, CNRS, LEO (FRE 2014), Rue de Blois, 45067 Orléans. E-mail: christophe.hurlin@univorleans.fr

ฯUniv. Orléans, CNRS, LEO (FRE 2014), Rue de Blois, 45067 Orléans. E-mail: sessi.tokpavi@univorleans.fr 


\section{Introduction}

Many authors have highlighted the extraordinary power of machine learning allied with economists' knowledge base to address real-world business and policy problems. See, for instance, Varian (2014), Mullainathan and Spiess (2017), Athey (2018), Charpentier et al. (2018), and Athey and Imbens (2019). In this article, we propose to combine the best of both worlds, namely, econometrics and machine learning, within the specific context of credit scoring. ${ }^{1}$ Our objective is to improve the predictive power of logistic regression models via feature engineering based on machine learning classifiers and penalisation techniques while keeping the model easily interpretable. Thus, our approach aims to propose a credit scoring methodology that avoids the perennial trade-off between interpretability and forecasting performance.

The use of econometric models for credit scoring dates back to the 1960s, when the credit card business arose and an automatised decision process was required. ${ }^{2}$ After a period of rather slow acceptance, credit scoring had, by the 1970s, become widely used by most banks and other lenders, and various econometric models were considered, including discriminant analysis (Altman, 1968), proportional hazard (Stepanova and Thomas, 2001), probit or logistic regression models (Steenackers and Goovaerts, 1989), among many others. Then, logistic regression gradually became the standard scoring model in the credit industry, mainly because of its simplicity and intrinsic interpretability. Most international banks still use this statistical model, especially for regulatory scores used to estimate the probability of default for capital requirements (Basel III) or for point-in-time estimates of expected credit losses (IFRS9).

Credit scoring was one of the first fields of application of machine learning techniques in economics. Some early examples are decision trees (Makowski, 1985; Coffman, 1986; Srinivasan and Kim, 1987), k-nearest neighbours (Henley and Hand, 1996, 1997), neural networks (NN) (Tam and Kiang, 1992; Desai et al., 1996; West, 2000; Yobas et al., 2000), and support vector machines (SVMs) (Baesens et al., 2003). At that time, the accuracy gains (compared to the standard logistic regression model) for creditworthiness assessment appeared to be limited (see the early surveys of Thomas, 2000 and Baesens et al., 2003). However, the performance of machine learning-based scoring models has been improved substantially since the adoption of ensemble (aggregation) methods, especially bagging and boosting methods (Finlay, 2011; Paleologo et al., 2010; Lessmann et al., 2015). ${ }^{3}$ In their

\footnotetext{
${ }^{1}$ Broadly defined, credit scoring is a statistical analysis that quantifies the level of credit risk associated with a prospective or current borrower.

${ }^{2}$ In a working paper of the National Bureau of Economic Research (NBER), Durand (1941) was the first to mention that the discriminant analysis technique, invented by Fisher in 1936, could be used to separate entities with good and bad credit.

${ }^{3}$ The ensemble or aggregation methods aim to improve the predictive performance of a given statistical or machine learning algorithm (weak learner) by using a linear combination (through averaging or majority vote) of predictions from many variants of this algorithm rather than a single prediction.
} 
extensive benchmarking study, Lessmann et al. (2015) compared 41 algorithms with various assessment criteria and several credit scoring datasets. They confirmed that the random forest method, i.e., the randomised version of bagged decision trees (Breiman, 2001), largely outperforms logistic regression and has progressively become one of the standard models in the credit scoring industry (Grennepois et al., 2018). Over the last decade, machine learning techniques have been increasingly used by banks and fintechs as challenger models (ACPR, 2020) or sometimes for credit production, generally associated with "new" data (social or communication networks, digital footprint, etc.) and/or "big data" (Hurlin and Pérignon, 2019). ${ }^{4}$

However, one of the main limitations of machine learning methods in the credit scoring industry comes from their lack of explainability and interpretability. ${ }^{5}$ Most of these algorithms, in particular ensemble methods, are considered "black boxes" in the sense that the corresponding scorecards and credit approval process cannot be easily explained to customers and regulators. This is consistent with financial regulators' current concerns about the governance of AI and the need for interpretability, especially in the credit scoring industry. See, for instance, the recent reports on this topic published by the French regulatory supervisor (ACPR, 2020), the Bank of England (Bracke et al., 2019), the European Commission (EC, 2020), and the European Banking Authority (EBA, 2020), among many others.

Within this context, we propose a hybrid approach called the penalised logistic tree regression model (hereafter PLTR). PLTR aims to improve the predictive performance of the logistic regression model through data pre-processing and feature engineering based on short-depth decision trees and a penalised estimation method while preserving the intrinsic interpretability of the scoring model. Formally, PLTR consists of a simple logistic regression model (econometric model side) including predictors extracted from decision trees (machine learning side). These predictors are binary rules (leaves) outputted by short-depth decision trees built with pairs of original predictive variables. To handle a possibly large number of decision-tree rules, we incorporate variable selection in the estimation through an adaptive lasso logistic regression model (Zou, 2006; Friedman et al., 2010), i.e., a penalised version of classic logistic regression.

The PLTR model has several advantages. First, it allows us to capture non-linear effects (i.e., thresholds and interactions between the features) that can arise in credit scoring data. It is recognised that ensemble methods consistently outperform logistic regression because the latter fails to fit these non-linear effects. For instance, the random forest method benefits from the recursive partitioning underlying decision trees and hence, by design, ac-

\footnotetext{
${ }^{4}$ See Óskarsdóttir et al. (2019) or Frost et al. (2019) for a general discussion about the value of big data for credit scoring. In the present article, we limit ourselves to the use of machine learning algorithms with "traditional data" for credit risk analysis.

${ }^{5}$ We do not distinguish explainability from interpretability.
} 
commodates unobserved multivariate threshold effects. The notable aspect of our approach consists of using these algorithms to pre-treat the predictors instead of modelling the default probability directly with machine learning classification algorithms. Second, PLTR provides parsimonious and interpretable scoring rules (e.g., marginal effects or scorecards) as recommended by the regulators, since it preserves the intrinsic interpretability of the logistic regression model and is based on a simple feature selection method.

In this article, we propose several Monte Carlo experiments to illustrate the inability of standard parametric models, i.e., standard logistic regression models with linear specification of the index or with quadratic and interaction terms, to capture well the non-linear effects (thresholds and interactions) that can arise in credit scoring data. Furthermore, these simulations allow us to evaluate the relative performance of PLTR in the presence of non-linear effects while controlling for the number of predictors. We show that PLTR outperforms standard logistic regression in terms of forecasting accuracy. Moreover, it compares competitively to the random forest method while providing an interpretable scoring function. We apply PLTR and six other benchmark credit scoring methodologies (random forest, linear logistic regression, non-linear logistic regression, non-linear logistic regression with adaptive lasso, an SVM and an NN) on four real credit scoring datasets. The empirical results confirm those obtained through simulations, as PLTR yields good forecasting performance for all the datasets. This conclusion is robust to the various predictive accuracy indicators considered by Lessmann et al. (2015). Finally, we show that PLTR also leads to more cost reductions than alternative credit scoring models.

Our paper contributes to the literature on credit scoring on various issues. First, our approach avoids the traditional trade-off between interpretability and forecasting performance. We propose here to restrict the intrinsic complexity of credit-score models rather than apply ex post interpretability methods to analyse the scoring model after training. Indeed, many model-agnostic methods have been recently proposed to make the "black box" machine learning models explainable and/or their decisions interpretable (see Molnar, 2019 for an overview). We can cite here among many others the partial dependence (PDP) or individual conditional expectation (ICE) plots, global or local (such as the LIME) surrogate models, feature interaction, Shapley values, Shapley additive explanations (SHAPE), etc. In the context of credit scoring models, Bracke et al. (2019) and Grennepois and Robin (2019) promoted the use of Shapley values. ${ }^{6}$ Bussman et al. (2019) recently proposed an explainable machine learning model specifically designed for credit risk management. Their model applies similarity networks to Shapley values so that the predictions are grouped according to the similarity in the underlying explanatory variables. However, obtaining the Shapley values requires considerable computing time because the number of coalitions

\footnotetext{
${ }^{6}$ This method assumes that each feature of an individual is a player in a game where its predictive abilities determine the pay-out of each feature (Lundberg and Lee, 2017).
} 
grows exponentially with the number of predictive variables, and computational shortcuts provide only approximate and unstable solutions. An alternative approach is the InTrees method proposed by Deng (2019). That algorithm extracts, measures, prunes, selects, and summarises rules from a tree ensemble and calculates frequent variable interactions. This helps detect simple decision rules from the forest that are important in predicting the outcome variable. Nevertheless, the algorithms underlying the extraction of these rules are not easy to disclose. Finally, our contribution can also be related to the methods designed to enable NNs and SVMs to be interpretable, especially the TREPAN (Thomas et al., 2017), Re-RX (Setiono et al., 2008), or ALBA (Martens et al., 2008) algorithms. However, there is a slight difference between these approaches and ours. While the latter aim to enable a model (i.e., NNs or SVMs) to be explainable/interpretable, PLTR aims to improve the predictive performance of a simple model (i.e., the logistic regression model) that is inherently interpretable.

Second, our approach can be viewed as a systematisation of common practises in the credit industry, where standard logistic regression is still the standard scoring model, especially for regulatory purposes. Indeed, credit risk modellers usually introduce non-linear effects in logistic regression by using ad hoc or heuristic pre-treatments and feature engineering methods (Hurlin and Pérignon, 2019) such as discretisation of continuous variables, merger of categories, and identification of non-linear effects with cross-product variables. In contrast, we propose here a systematic and automatic approach for modelling such unobserved non-linear effects based on short-depth decision trees. Thus, PLTR may allow model developers to significantly reduce the time spent on data management and data pre-processing steps.

More generally, our paper complements the literature devoted to hybrid classification algorithms. The PLTR model differs from the so-called logit-tree models, i.e., trees that contain logistic regressions at the leaf nodes such as the logistic tree with unbiased selection (LOTUS) in Chan and Loh (2004) and the logistic model tree (LMT) in Landwehr et al. (2005). Although similar in spirit, our PLTR method also contrasts with the hybrid CARTlogit model of Cardell and Steinberg (1998). Indeed, to introduce multivariate threshold effects in logistic regression, Cardell and Steinberg (1998) used a single non-pruned decision tree. However, the large depth of this unique tree complicates interpretability and may lead to predictor inflation that is not controlled for (e.g., through penalisation, as in our case). PLTR also shares similarities with the two-step classification algorithm recently proposed by De Caigny et al. (2018) in the context of customer churn prediction. Their initial analysis consisted of applying a decision tree to split customers into homogeneous segments corresponding to the leaves of the decision tree, while the second step consisted of estimating a logistic regression model for each segment. However, their method is based on a single non-pruned decision tree as in the hybrid CART-logit model. Furthermore, their 
objective was to improve the predictive performance of the logistic regression by identifying homogeneous subsets of customers and not by introducing non-linear effects as in the PLTR approach.

The rest of the article is structured as follows. Section 2 analyses the performance of logistic regression and random forest in the presence of univariate and multivariate threshold effects through Monte Carlo simulations. In Section 3, we introduce the PLTR credit scoring method and assess through Monte Carlo simulations its accuracy and interpretability (parsimony) in the presence of threshold effects. Section 4 describes an empirical application with a benchmark dataset. The robustness of the results to dataset choice is explored in Section 5. Section 6 compares the models from an economic point of view, while the last section concludes the paper.

\section{Threshold effects in logistic regression}

\subsection{Non-linear effects and the logistic regression model}

Let $\left(x_{i}, y_{i}\right), i=1, \ldots, n$ be a sample of size $n$ of independent and identically distributed observations, where $x_{i} \in \mathbb{R}^{p}$ is a $p$-dimensional vector of predictors and $y_{i} \in\{0,1\}$ is a binary variable taking the value one when the i-th borrower defaults and zero otherwise. The goal of a credit scoring model is to provide an estimate of the posterior probability $\operatorname{Pr}\left(y_{i}=1 \mid x_{i}\right)$ that borrower $i$ defaults given its attributes $x_{i}$. The relevant characteristics of the borrower vary according to its status: household or company. For corporate credit risk scoring, the candidate predictive variables $x_{i, j}, j=1, \ldots, p$, may include balance-sheet financial variables that cover various aspects of the financial strength of the firm, such as the firm's operational performance, its liquidity, and capital structure (Altman, 1968). For instance, using a sample of 4,796 Belgian firms, Bauweraerts (2016) shows the importance of taking into account the level of liquidity, solvency and profitability of the firm in forecasting its bankruptcy risk. For small and medium enterprises (SMEs), specific variables related to the financial strength of the firm's owner are also shown to be important (Wang, 2012). For retail loans, financial variables such as the number and amount of personal loans, normal repayment frequency of loans, the number of credit cards, the average overdue duration of credit cards and the amount of housing loans are combined with socio-demographic factors. A typical example is the FICO score, which is widely used in the US financial industry to assess the creditworthiness of individual customers.

Regardless of the type of borrower, the conditional probability of default is generally modelled using a logistic regression with the following specification:

$$
\operatorname{Pr}\left(y_{i}=1 \mid x_{i}\right)=F\left(\eta\left(x_{i} ; \beta\right)\right)=\frac{1}{1+\exp \left(-\eta\left(x_{i} ; \beta\right)\right)},
$$

where $F($.$) is the logistic cumulative distribution function and \eta\left(x_{i} ; \beta\right)$ is the so-called index 
function defined as

$$
\eta\left(x_{i} ; \beta\right)=\beta_{0}+\sum_{j=1}^{p} \beta_{j} x_{i, j}
$$

where $\beta=\left(\beta_{0}, \beta_{1}, \ldots, \beta_{p}\right) \in \mathbb{R}^{p+1}$ is an unknown vector of parameters. The estimator $\widehat{\beta}$ is obtained by maximizing the log-likelihood function

$$
\mathcal{L}\left(y_{i} ; \beta\right)=\sum_{i=1}^{n}\left\{y_{i} \log \left\{F\left(\eta\left(x_{i} ; \beta\right)\right)\right\}+\left(1-y_{i}\right) \log \left\{1-F\left(\eta\left(x_{i} ; \beta\right)\right)\right\}\right\}
$$

The main advantage of the logistic regression model is its simple interpretation. Indeed, this model searches for a single linear decision boundary in the predictors' space. The core assumption for finding this boundary is that the index $\eta\left(x_{i} ; \beta\right)$ is linearly related to the predictive variables. In this framework, it is easy to evaluate the relative contribution of each predictor to the probability of default. This is achieved by computing marginal effects as

$$
\frac{\partial \operatorname{Pr}\left(y_{i}=1 \mid x_{i}\right)}{\partial x_{i, j}}=\beta_{j} \frac{\exp \left(\eta\left(x_{i} ; \beta\right)\right)}{\left[1+\exp \left(\eta\left(x_{i} ; \beta\right)\right)\right]^{2}}
$$

with estimates obtained by replacing $\beta$ with $\widehat{\beta}$. Thus, a predictive variable with a positive (negative) significant coefficient has a positive (negative) impact on the borrower's default probability.

This simplicity comes at a cost when significant non-linear relationships exist between the default indicator, $y_{i}$, and the predictive variables, $x_{i}$. A very common type of non-linearity can arise from the existence of a univariate threshold effect on a single predictive variable, but it can also be generalised to a combination of such effects (multivariate threshold effects) across variables. A typical example of the former case in the context of credit scoring is the income "threshold effect", which implies the existence of an endogenous income threshold below (above) which default probability is more (less) prominent. The income threshold effect can obviously interact with other threshold effects, leading to highly non-linear multivariate threshold effects. The common practise to approximate non-linear effects in credit scoring applications is to introduce quadratic and interaction terms in the index function $\eta\left(x_{i} ; \beta\right)$. However, such a practise is not successful when unobserved threshold effects are at stake.

To illustrate the inability of standard parametric models, i.e., standard logistic regression model or logistic regression with quadratic and interaction terms, to capture accurately the non-linear effects (thresholds and interactions) that can arise in credit scoring data, we propose a Monte Carlo simulation experiment. In a first step (simulation step), we generate $p$ predictive variables $x_{i, j}, j=1, \ldots, p, i=1, \ldots, n$, where the sample size is set to $n=5,000$. Each predictive variable $x_{i, j}$ is assumed to follow the standard Gaussian distribution. The 
index function $\eta\left(x_{i} ; \Theta\right)$ is simulated as follows:

$$
\eta\left(x_{i} ; \Theta\right)=\beta_{0}+\sum_{j=1}^{p} \beta_{j} \mathbf{1}\left(x_{i, j} \leq \gamma_{j}\right)+\sum_{j=1}^{p-1} \sum_{k=j+1}^{p} \beta_{j, k} \mathbf{1}\left(x_{i, j} \leq \delta_{j}\right) \mathbf{1}\left(x_{i, k} \leq \delta_{k}\right),
$$

where $\mathbf{1}($.$) is the indicator function and \Theta=\left(\beta_{0}, \beta_{1}, \ldots, \beta_{p}, \beta_{1,2}, \ldots, \beta_{p-1, p}\right)^{\prime}$ is the vector of parameters, with each component randomly drawn from a uniform $[-1,1]$ distribution, and $\left(\gamma_{1}, \ldots, \gamma_{p}, \delta_{1}, \ldots, \delta_{p}\right)^{\prime}$ are some threshold parameters, whose values are randomly selected from the support of each predictive variable generated while excluding data below (above) the first (last) decile. The default probability is then obtained for each individual by plugging (2) into (1). Subsequently, the simulated target binary variable $y_{i}$ is obtained as

$$
y_{i}= \begin{cases}1 & \text { if } \operatorname{Pr}\left(y_{i}=1 \mid x_{i}\right)>\pi \\ 0 & \text { otherwise }\end{cases}
$$

where $\pi$ stands for the median value of the generated probabilities.

In a second step (estimation step), we estimate by maximum likelihood two logistic regressions on the simulated data $\left\{y_{i}, x_{i}\right\}_{i=1}^{n}:(i)$ a standard logistic regression model and (ii) a (non-linear) logistic regression with quadratic and interaction terms. For the standard logistic regression model, the conditional probability is based on a linear index defined as

$$
\eta\left(x_{i} ; \beta\right)=\beta_{0}+\sum_{j=1}^{p} \beta_{j} x_{i, j} .
$$

For non-linear logistic regression, we also include quadratic and interaction terms

$$
\eta^{(n l)}\left(x_{i} ; \Theta^{(n l)}\right)=\alpha_{0}+\sum_{j=1}^{p} \alpha_{j} x_{i, j}+\sum_{j=1}^{p} \xi_{j} x_{i, j}^{2}+\sum_{j=1}^{p-1} \sum_{k=j+1}^{p} \zeta_{j, k} x_{i, j} x_{i, k} .
$$

where $\Theta^{(n l)}=\left(\alpha_{0}, \alpha_{1}, \ldots, \alpha_{p}, \xi_{1}, \ldots, \xi_{p}, \zeta_{1,2}, \ldots, \zeta_{p-1, p}\right)^{\prime}$ is the unknown vector of parameters.

Figure 1 displays the average value of the percent of correct classification (PCC) values of these two models over 100 simulations and for different numbers of predictors $p=4, \ldots, 20 .^{7}$ We observe that their accuracy decreases with the number of predictors. This result arises because the two logistic regression models are misspecified because they do not control for threshold and interaction effects, and their degree of misspecification increases with additional predictors. Indeed, in our DGP (i.e., Equation 2), the number of regressors controls for the degree of non-linearity of the data generating process: more predictors correspond to more threshold and interaction effects. These results suggest that in the presence of univariate and bivariate threshold effects involving many variables, logistic regression with a linear index function, eventually augmented with quadratic and interaction terms, fails to discriminate between good and bad loans. In the case where $p=20$, the PCCs of the logistic regression models are equal to only $72.30 \%$ and $75.19 \%$.

\footnotetext{
${ }^{7}$ We divide the simulated sample into two sub-samples of equal size at each replication. The training sample is used to estimate the parameters of the logistic regression model, while the classification performance is evaluated on the test sample. To compute the PCC, we estimate $y_{i}$ by comparing the estimated probabilities of default, $\widehat{p}_{i}$, to an endogenous threshold $\widehat{\pi}$. As usual in the literature, we set $\widehat{\pi}$ to a value such that the number of predicted defaults in the learning sample is equal to the observed number of defaults.
} 


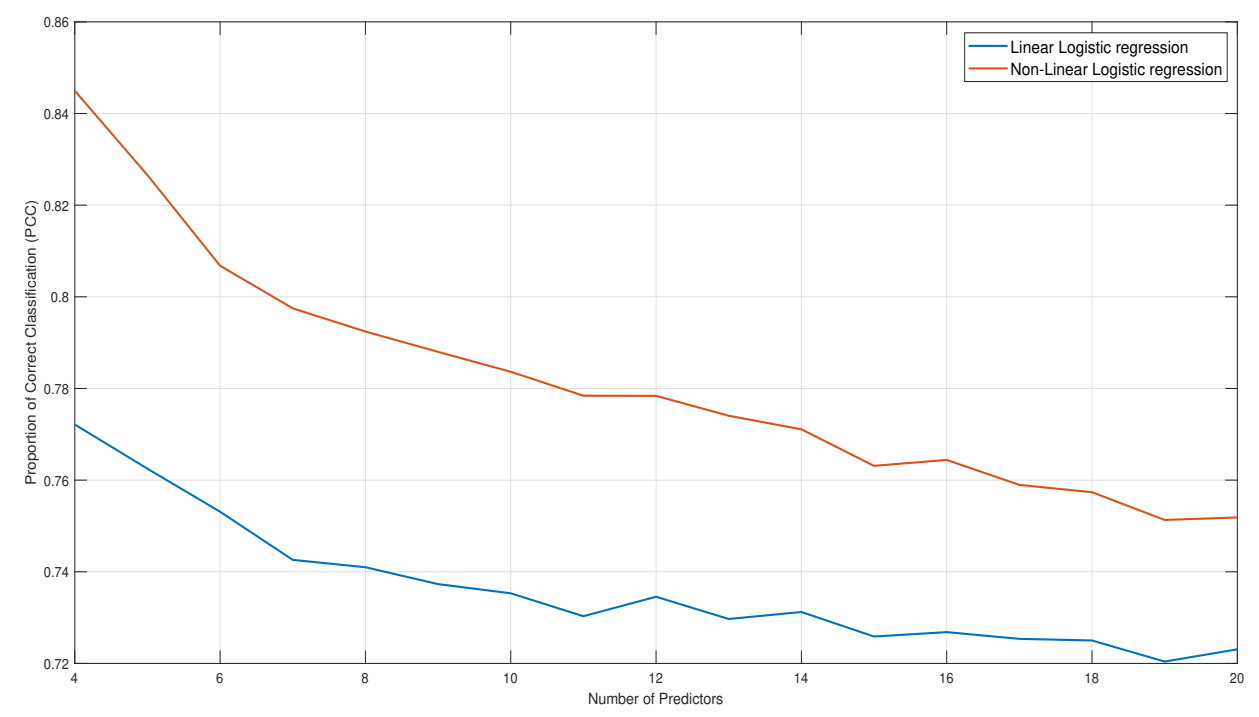

Figure 1: Comparison of performances under univariate and bivariate threshold effects: linear and non-linear logistic regressions

\subsection{Machine learning for non-linear effects}

In the context of credit scoring, ensemble methods based on decision trees, such as the random forest method, provide better classification performance than standard logistic regression models (Paleologo et al., 2010; Finlay, 2011; Lessmann et al., 2015). The outperformance of the random forest method arises from the non-linear "if-then-else" rules underlying decision trees. ${ }^{8}$ Formally, for a given tree, $l$, the algorithm proceeds as follows. Let $\mathcal{D}_{m, l}$ be the data (sub)set at a given node $m$ of this tree. We denote by $\theta_{m, l}=\left(j_{m, l}, t_{m, l, j}\right)$ a candidate split, where $j_{m, l}=1, \ldots, p$ indicates a given predictor and $t_{m, l, j}$ is a threshold value in the support of this variable. The algorithm partitions the data $\mathcal{D}_{m, l}$ into two subsets $\mathcal{D}_{m, l, 1}\left(\theta_{m, l}\right)$ and $\mathcal{D}_{m, l, 2}\left(\theta_{m, l}\right)$, with $^{9}$

$$
\begin{aligned}
& \mathcal{D}_{m, l, 1}\left(\theta_{m, l}\right)=\left(x_{i}, y_{i}\right) \mid x_{i, j}<t_{m, l, j}, \\
& \mathcal{D}_{m, l, 2}\left(\theta_{m, l}\right)=\left(x_{i}, y_{i}\right) \mid x_{i, j} \geq t_{m, l, j},
\end{aligned}
$$

where the parameter estimates $\widehat{\theta}_{m, l}$ satisfy

$$
\widehat{\theta}_{m, l}=\left(\widehat{j}_{m, l}, \widehat{t}_{m, l, j}\right)=\underset{\theta_{m, l}}{\arg \max } \mathcal{H}\left(\mathcal{D}_{m, l}\right)-\frac{1}{2}\left(\mathcal{H}\left(\mathcal{D}_{m, l, 1}\left(\theta_{m, l}\right)\right)+\mathcal{H}\left(\mathcal{D}_{m, l, 2}\left(\theta_{m, l}\right)\right)\right)
$$

with $\mathcal{H}($.$) a measure of diversity, e.g., the Gini criterion, applied to the full sample and$ averaged across the two sub-samples. Hence, $\widehat{\theta}_{m, l}$ appears as the value of $\theta_{m, l}$ that reduces

\footnotetext{
${ }^{8}$ Indeed, the latter is a non-parametric supervised learning method based on a divide-and-conquer greedy algorithm that recursively partitions the training sample into smaller subsets to group together as accurately as possible individuals with the same behaviour, i.e., the same value of the binary target variable " $y_{i} "$.

${ }^{9}$ To simplify the description of the algorithm, we focus only on quantitative predictors. A similar procedure is available for qualitative predictors.
} 
diversity the most within each subset resulting from the split. The splitting process is repeated until the terminal sub-samples, also known as leaf nodes, contain homogeneous individuals according to a predefined homogeneity rule. We denote by $M_{l}$ the total number of splits in tree $l$ and by $\left|T_{l}\right|$ the corresponding number of leaf nodes.

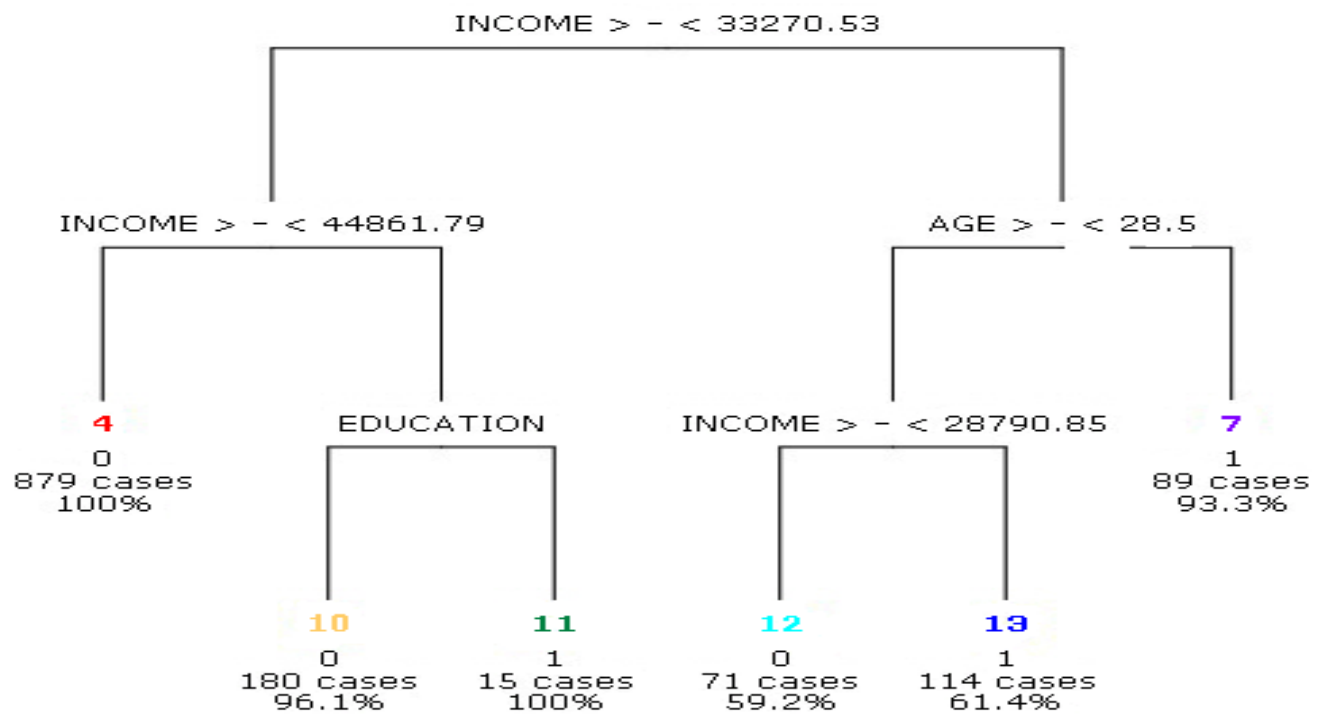

Figure 2: Example of a decision tree for credit scoring

An illustrative example of a decision tree is given below in Figure 2. At the first iteration (or split), $m=1, \widehat{\theta}_{m, l}$ is defined by $\left(\widehat{j}_{m, l}, \widehat{t}_{m, l, 1}\right)$, with $\widehat{j}_{m, l}$ the index of the variable "income" and $\widehat{t}_{m, l, 1}=33270.53$. The other iterations also include "age" and "education" for further refinements. The process ends with a total number of 5 splits and 6 leaf nodes labelled $10,11,12,13,4$ and 7 . Each leaf node $\mathcal{R}_{t}, t=1, \ldots,\left|T_{l}\right|$ includes a specific proportion of individuals belonging to each class of borrowers ( $1=$ "default", $0=$ "no default"). For instance, leaf node " 7 " contains 89 individuals, $93.3 \%$ of them having experienced a default event. Note that each of these individuals has an income lower than 33270.53 and is less than 28.5 years old. The predominant class in each leaf defines the predicted value of $y_{i}$ for individuals $i$ that belong to that particular leaf. Formally, the predicted default value for the $i^{\text {th }}$ individual is

$$
h_{l}\left(x_{i} ; \widehat{\Theta}_{l}\right)=\sum_{t=1}^{\left|T_{l}\right|} c_{t} \mathcal{R}_{i, t},
$$

where $\Theta_{l}=\left(\theta_{m, l}, m=1, \ldots, M_{l}\right)$ is the parameter vector for tree $l, \mathcal{R}_{i, t}=1_{\left(i \in \mathcal{R}_{t}\right)}$ indicates whether individual $i$ belongs to leaf $\mathcal{R}_{t}$, and $c_{t}$ is the dominant class of borrowers in that leaf node. For example, in leaf node 7 , the "default" class is dominant; hence, the predicted value $h_{l}\left(x_{i}\right)$ is equal to 1 for all the individuals that belong to this leaf node. Note that this simple tree allows us to identify both interaction and threshold effects. For instance, in the simple example of Figure 2, the predicted value can be viewed as the result of a kind 
of linear regression ${ }^{10}$ on the product of two binary variables that take a value of one if the income is lower than 33270.53 and the age is less than 28.5.

The random forest method is a bagging procedure that aggregates many uncorrelated decision trees. It exploits decision-tree power to detect univariate and multivariate threshold effects while reducing their instability. Its superior predictive performance springs from the variance reduction effect of bootstrap aggregation for non-correlated predictions (Breiman, 1996). Let $L$ trees be constructed from bootstrap samples (with replacement) of fixed size drawn from the original sample. To ensure a low level of correlation among those trees, the random forest algorithm chooses the candidate variable for each split in every tree, $j_{m, l}$ with $m \in\left\{1, \ldots, M_{l}\right\}$ and $l \in\{1, \ldots, L\}$, from a restricted number of randomly selected predictors among the $p$ available ones. The default prediction of the random forest for each borrower, $h\left(x_{i}\right)$, is obtained by the principle of majority vote; that is, $h\left(x_{i}\right)$ corresponds to the mode of the empirical distribution of $h_{l}\left(x_{i} ; \widehat{\Theta}_{l}\right), l=1, \ldots, L$.

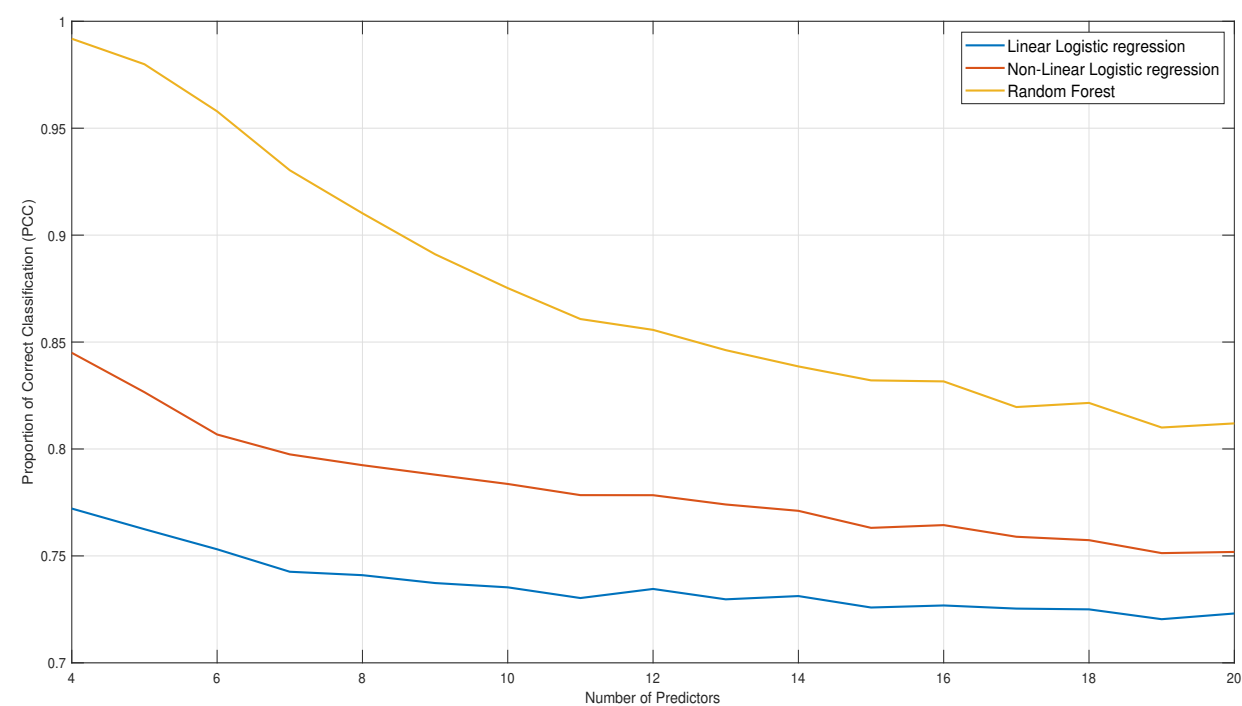

Figure 3: Comparison of performances under univariate and bivariate threshold effects: linear and non-linear logistic regressions and the random forest method

To illustrate the ability of the random forest method to capture the non-linear effects that can arise in credit scoring data well, we consider the same Monte Carlo framework as in Section 2.1. The proportion of correct classification for the random forest algorithm, displayed as a yellow line in Figure 3, is computed over the same test samples of length 2, 500 as the PCCs of the logistic regressions previously discussed. The optimal number of trees in the forest, $L$, is tuned using the out-of-bag error. Our results confirm the empirical findings of the literature: in the presence of non-linear effects, random forest outperforms not only linear logistic regression (as expected) but also non-linear logistic regression. This

\footnotetext{
${ }^{10}$ This equivalence is true only in the case of a regression tree when the target variable $y$ is continuous.
} 
illustrates the ability of random forests to capture both threshold and interaction effects between the predictors well. These findings are valid regardless of the number of predictors, even if the differences in classification performance between the three models are decreasing in the number of predictors. Indeed, as the number of predictors increases, the complexity and the non-linearity of the DGP also increases, which diminishes the performance of all the classifiers. For instance, the PPCs are equal to $99.18 \%$ (resp. 84.50\%) for the random forest (resp. logistic regression with quadratic and interaction terms) in the case with 4 predictors, against $81.20 \%$ (resp. $75.19 \%$ ) in the case with 20 predictors.

Despite ensuring good performance, the aggregation rule (majority vote) underlying the random forest method leads to a prediction rule that lacks interpretation. This opacity is harmful for credit scoring applications, where decision makers and regulators need simple and interpretable scores (see ACPR, 2020 and EC, 2020, among many others). The key question here is how to find a suitable trade-off between predictive performance and interpretability. To address this issue, two lines of research can be explored. First, one can try to diminish the complexity of the random forest method's aggregation rule by selecting (via an objective criterion) only some trees or decision rules in the forest. ${ }^{11}$ Second, we can preserve the simplicity of logistic regression while improving its predictive performance with univariate and bivariate endogenous threshold effects. We opt here for the second line of research, with the PLTR hybrid scoring approach.

\section{Penalised logistic tree regression}

\subsection{Description of the methodology}

PLTR aims to improve the predictive performance of the logistic regression model through new predictors based on short-depth decision trees and a penalised estimation method while preserving the intrinsic interpretability of the scoring model. The algorithm proceeds in two steps.

The objective of the first step is to identify threshold effects from trees with two splits. For illustration, take the income and age as the $j^{\text {th }}$ and $k^{\text {th }}$ explanatory variables, respectively, and assume that income is more informative than age in explaining credit default. For each individual $i$, the corresponding decision tree generates three binary variables, each associated with a terminal node. The first binary variable $\mathcal{V}_{i, 1}^{(j)}$ accounts for univariate threshold effects and takes the value of one when the income of individual $i$ is higher than an estimated income threshold and zero otherwise. The second (third) binary variable $\mathcal{V}_{i, 2}^{(j, k)}$ $\left(\mathcal{V}_{i, 3}^{(j, k)}\right)$, representing bivariate threshold effects, is equal to one when the person's income is lower than its threshold and at the same time his/her age is lower (higher) than an estimated

\footnotetext{
${ }^{11}$ Note that this is the approach underlying the so-called InTrees method of Deng (2019), who proposed a methodology to render the random forest outputs interpretable by extracting simple rules from a tree ensemble.
} 
age threshold and zero otherwise. ${ }^{12}$ Note that this particular form of splitting should arise when both variables are informative, i.e., each of them is selected in the iterative process of splitting. If the second variable is non-informative (age), the tree relies twice on the first informative variable (income). Figure 4 gives an illustration of the splitting process.

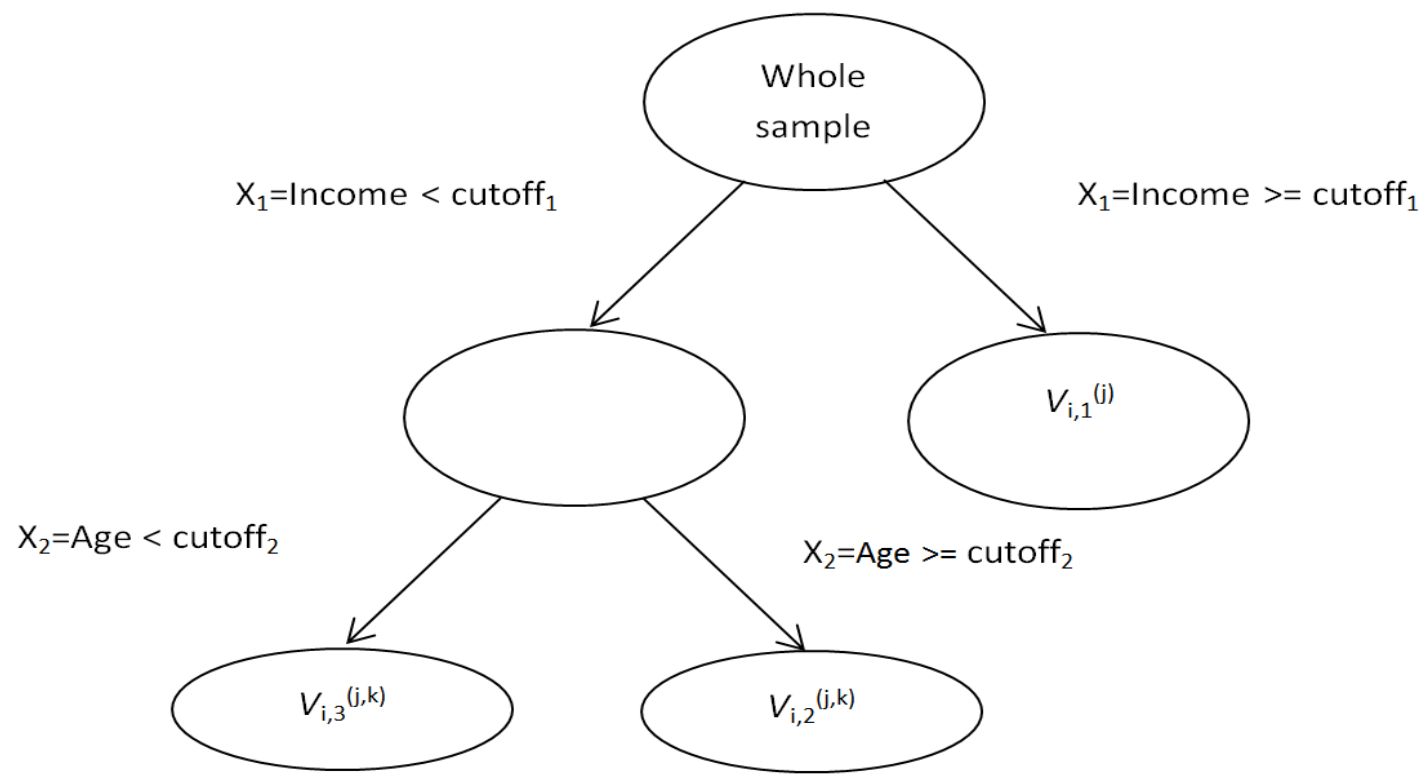

Figure 4: Illustration of the two-stage splitting process

One leaf of each of the two branches originating from the root of the tree is retained to cover both one and two splits, i.e., the first two binary variables $\mathcal{V}_{i, 1}^{(j)}$ and $\mathcal{V}_{i, 2}^{(j, k)}$ in the example above. We count at most $p+q$ threshold effects for inclusion in our logistic regression, where $p$ represents the number of predictive variables and $q$ denotes the total number of couples of predictors, with $q \leq p \times(p-1) / 2$. This is the case because the univariate threshold effects $\mathcal{V}_{i, 1}^{(j)}$ are generated only by the variables retained in the first split irrespective of the variables retained in the second split. Some predictive variables may be selected in the first split of several trees, while others may never be retained. The latter group does not produce any univariate threshold effects, while the former group delivers identical univariate threshold effects, $\mathcal{V}_{i, 1}^{(j)}$, out of which only one is included in the logistic regression. ${ }^{13}$

In the second step, the endogenous univariate and bivariate threshold effects previously

\footnotetext{
${ }^{12}$ It is also possible that the univariate threshold variable $\mathcal{V}_{i, 1}^{(j)}$ takes the value of one when the income is lower than an estimated income threshold, and zero otherwise. In that case, the bivariate threshold effect $\mathcal{V}_{i, 2}^{(j, k)}\left(\mathcal{V}_{i, 3}^{(j, k)}\right)$ is equal to one when the individual's income is higher than its threshold and at the same time his/her age is lower (higher) than an estimated age threshold, and zero otherwise.

${ }^{13}$ Note that one could also go beyond two splits by analysing triplets or quadruplets of predictive variables. Such a procedure would allow the inclusion of more complex non-linear relationships in the logistic regression. Nevertheless, the expected uprise in performance would come at the cost of increased complexity of the model toward that of random forests, which would plunge its level of interpretability. For this reason, in our PLTR model, we use only short-depth decision trees involving two splits.
} 
obtained are plugged in the logistic regression

$$
\operatorname{Pr}\left(y_{i}=1 \mid \mathcal{V}_{i, 1}^{(j)}, \mathcal{V}_{i, 2}^{(j, k)} ; \Theta\right)=\frac{1}{1+\exp \left[-\eta\left(\mathcal{V}_{i, 1}^{(j)}, \mathcal{V}_{i, 2}^{(j, k)} ; \Theta\right)\right]},
$$

with

$$
\eta\left(\mathcal{V}_{i, 1}^{(j)}, \mathcal{V}_{i, 2}^{(j, k)} ; \Theta\right)=\beta_{0}+\sum_{j=1}^{p} \alpha_{j} x_{i}+\sum_{j=1}^{p} \beta_{j} \mathcal{V}_{i, 1}^{(j)}+\sum_{j=1}^{p-1} \sum_{k=j+1}^{p} \gamma_{j, k} \mathcal{V}_{i, 2}^{(j, k)}
$$

the index and $\Theta=\left(\beta_{0}, \alpha_{1}, \ldots, \alpha_{p}, \beta_{1}, \ldots, \beta_{p}, \gamma_{1,2}, \ldots, \gamma_{p-1, p}\right)^{\prime}$ the set of parameters to be estimated. The corresponding log-likelihood is

$$
\begin{aligned}
\mathcal{L}\left(\mathcal{V}_{i, 1}^{(j)}, \mathcal{V}_{i, 2}^{(j, k)} ; \Theta\right)= & \frac{1}{n} \sum_{i=1}^{n}\left[y_{i} \log \left[F\left(\eta\left(\mathcal{V}_{i, 1}^{(j)}, \mathcal{V}_{i, 2}^{(j, k)} ; \Theta\right)\right)\right]\right. \\
& \left.+\left(1-y_{i}\right) \log \left[1-F\left(\eta\left(\mathcal{V}_{i, 1}^{(j)}, \mathcal{V}_{i, 2}^{(j, k)} ; \Theta\right)\right)\right]\right],
\end{aligned}
$$

where $F\left(\eta\left(\mathcal{V}_{i, 1}^{(j)}, \mathcal{V}_{i, 2}^{(j, k)} ; \Theta\right)\right)$ is the logistic cdf. The estimate $\widehat{\Theta}$ is obtained by maximizing the above log-likelihood with respect to the unknown parameters $\Theta$. Note that the length of $\Theta$ depends on the number of predictive variables, $p$, which can be relatively high. For instance, there are 45 couples of variables when $p=10$; this leads to a maximum number of 55 univariate and bivariate threshold effects that play the role of predictors in our logistic regression.

To prevent overfitting issues in this context with a large number of predictors, a common approach is to rely on penalisation (regularisation) for both estimation and variable selection. In our case, this method consists of adding a penalty term to the negative value of the loglikelihood function, such that

$$
\mathcal{L}_{p}\left(\mathcal{V}_{i, 1}^{(j)}, \mathcal{V}_{i, 2}^{(j, k)} ; \Theta\right)=-\mathcal{L}\left(\mathcal{V}_{i, 1}^{(j)}, \mathcal{V}_{i, 2}^{(j, k)} ; \Theta\right)+\lambda P(\Theta)
$$

where $P(\Theta)$ is the additional penalty term and $\lambda$ is a tuning parameter that controls the intensity of the regularisation and which is selected in such a way that the resulting model minimises the out-of-sample error. The optimal value of the tuning parameter $\lambda$ is usually obtained by relying on a grid search with cross-validation or by using some information criteria. In addition, several penalty terms $P(\Theta)$ have been proposed in the related literature (Tibshirani, 1996; Zou and Hastie, 2005; Zou, 2006). Here, we consider the adaptive lasso estimator of Zou (2006). Note that the adaptive lasso satisfies the oracle property; i.e., the probability of excluding relevant variables and selecting irrelevant variables is zero, contrary to the standard lasso penalisation (Fan and Li, 2001). The corresponding penalty term is $P(\Theta)=\sum_{v=1}^{V} w_{v}\left|\theta_{v}\right|$ with $w_{v}=\left|\widehat{\theta}_{v}^{(0)}\right|^{-\nu}$, where $\widehat{\theta}_{v}^{(0)}, v=1, \ldots, V$, are consistent initial estimators of the parameters and $\nu$ is a positive constant. The adaptive lasso estimators are obtained as

$$
\widehat{\Theta}_{\text {alasso }}(\lambda)=\underset{\Theta}{\arg \min }-\mathcal{L}\left(\mathcal{V}_{i, 1}^{(j)}, \mathcal{V}_{i, 2}^{(j, k)} ; \Theta\right)+\lambda \sum_{v=1}^{V} w_{v}\left|\theta_{v}\right| .
$$


In practise, we set the parameter $\nu$ to 1 and the initial estimator $\widehat{\theta}_{j}^{(0)}$ to the value obtained from the logistic-ridge regression (Hoerl and Kennard, 1970), and the only free tuning parameter, $\lambda$, is found via 10 -fold cross-validation. ${ }^{14}$

In summary, PLTR is a hybrid classification model designed to increase the predictive power of the logistic regression model via feature engineering. Its first step consists of creating additional binary predictors based on short-depth decision trees built with couples of predictive variables. These binary variables are then introduced, in a second step, in a penalised logistic regression model, where the adaptive lasso is used for both estimation and variable selection.

\subsection{PLTR under threshold effects: Monte Carlo evidence}

In this subsection, we assess the accuracy and interpretability of the PLTR method in the presence of threshold effects. For that, we consider the same Monte Carlo experiment as that defined in Section 2.

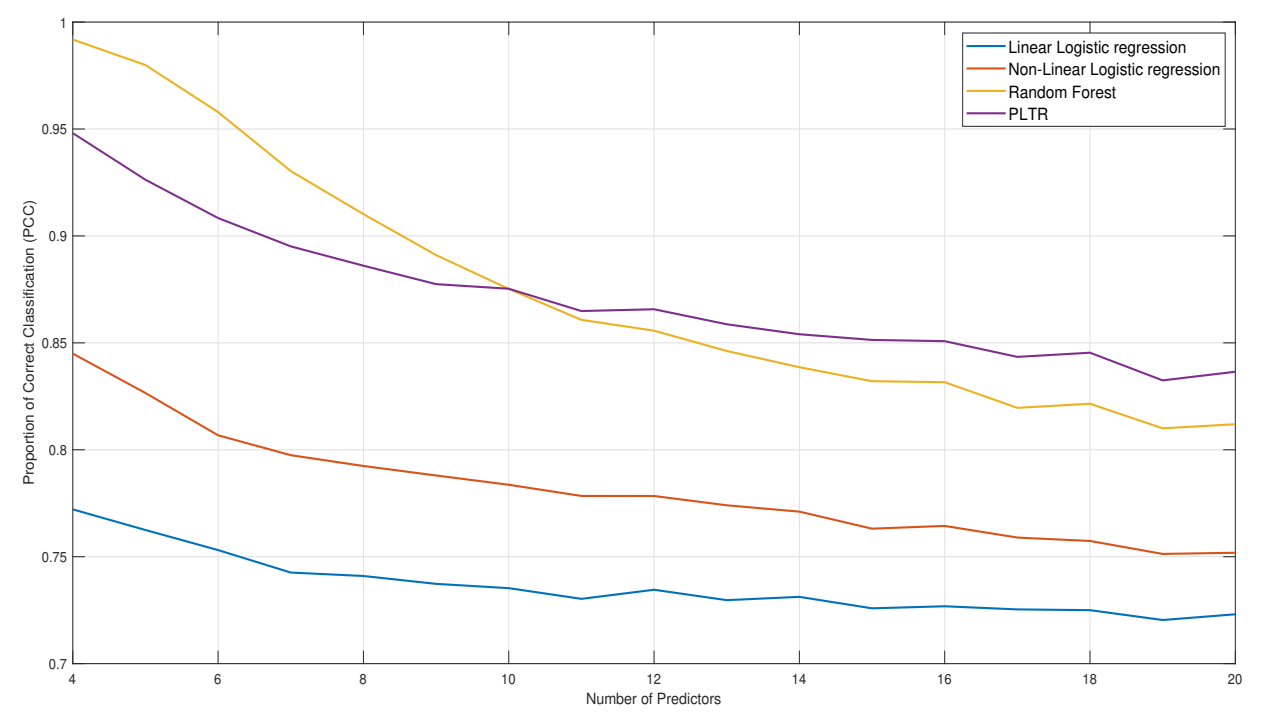

Figure 5: Comparison of performances under univariate and bivariate threshold effects: linear and non-linear logistic regressions, the random forest method and PLTR

Figure 5 displays the PCC for our PLTR method computed over the same test samples of length 2,500 that were generated with the DGP in (2)-(3). The main conclusion is that the PLTR method outperforms the two versions of the logistic regression, i.e., with and without

\footnotetext{
${ }^{14}$ Different estimation algorithms have been developed in the literature to estimate regression models with the adaptive lasso penalty (for a given value of $\lambda$ ): the quadratic programming technique (Shewchuk et al., 1994), the shooting algorithm (Zhang and Lu, 2007), the coordinate-descent algorithm (Friedman et al., 2010), and the Fisher scoring algorithm (Park and Hastie, 2007). Most of them are implemented in software such as MATLAB and R, and we rely here on the algorithm based on Fisher scoring. See McIlhagga (2016) for more details on this optimisation algorithm.
} 
quadratic and interaction terms. Equally important, when there are few predictors, i.e., $p$ is small, the PCC of PLTR is lower than that of random forest. However, as $p$ increases, the performance of PLTR approaches that of the random forest method, and both models have approximately the same classification performance. For example, the PCCs are equal to 94.81 for our new method and 99.18 for the random forest with $p=4$, against 83.65 and 81.20 for $p=20$, respectively. Note that the latter case seems more realistic, as credit scoring applications generally rely on a large set of predictors in practise.

Performance is not the only essential criterion for credit scoring managers. The other fundamental characteristic of a good scoring model is interpretability. Interpretability and accuracy are generally two competing objectives: the first is favoured by simple models, the latter by complex models. Moreover, the degree of interpretability of a credit scoring model is difficult to measure. As discussed in Molnar (2019), there is no real consensus in the literature about what is interpretable for machine learning, nor is it clear how to measure this factor. Doshi-Velez and Kim (2017) distinguishes three levels of evaluation of interpretability: the application level, the human level, and the function level. While the application and human levels are related to the understanding of the conclusion of a model (from an expert or a layperson, respectively), the function level corresponds to the evaluation of decision rules from a statistical viewpoint (for example, the depth of a decision tree). In the specific context of credit scoring, Bracke et al. (2019) distinguishes six different types of stakeholders (developers, 1st- and 2nd-line model checkers, management, regulators, etc. ). ${ }^{15}$ Each of them has its own definition of what interpretability should be and how to measure it. For instance, the developer and 1st-line checkers may be interested in individual predictions when they obtain customer queries and in better understanding outliers. In contrast, second-line model checkers, management, and prudential regulators are likely to adopt a more general viewpoint and may be less interested in individual predictions.

In the credit scoring context, interpretability can be measured from at least two perspectives. First, one can consider simple metrics such as the size of the set of decision rules. This indicator allows us to compare models in terms of ease of interpretation: the fewer the rules in a decision set, the easier it is for a user to understand all the conditions that correspond to a particular class label. The size of a given rule in a decision set is a complementary measure. Indeed, if the number of predicates in a rule is too large, it will lose its natural interpretability. This perspective corresponds to the function level evaluation mentioned by Doshi-Velez and Kim (2017). Second, one can interpret the decision rules through marginal

\footnotetext{
${ }^{15}$ Bracke et al. (2019) distinguished the (i) developers, i.e., those developing or implementing an ML application; (ii) 1st-line model checkers, i.e., those directly responsible for ensuring that model development is of sufficient quality; (iii) management responsible for the application; (iv) 2nd-line model checkers, i.e., staff that, as part of a firm's control functions, independently check the quality of model development and deployment; (v) conduct regulators that are interested in deployed models being in line with conduct rules and (vi) prudential regulators that are interested in deployed models being in line with prudential requirements.
} 
effects, elasticities, or scorecards. This second perspective corresponds to the human-level evaluation evoked by Doshi-Velez and Kim (2017) or to the global model interpretability defined by Molnar (2019). Which features are important and what kind of interactions take place between them?

In this paper, we confirm this trade-off between interpretability and classification performance. The less accurate model, i.e., the logistic regression model, is intrinsically interpretable through marginal effects or explicit scorecard. In contrast, the model with the highest classification performance among our competing models, i.e., the random forest model, is not interpretable for two reasons. First, the forest relies on many trees with many splits, which involves many complicated if-then-else rules. Second, the rules obtained from the trees are aggregated via the majority vote.

Within this context, our PLTR method is a parsimonious solution to the trade-off between performance and interpretability. The scoring decision rules are simple to interpret through marginal effects (as well as elasticities and scorecards) similar to those of traditional logistic regression. This is facilitated by the simple decision rules obtained in the first step of the procedure from short-depth decision trees. Indeed, the skeleton of our PLTR is actually a logistic regression model with binary indicators that account for endogenous univariate and bivariate threshold effects. The complete loan-decision process based on the PLTR method is illustrated in Figure 6. The input of the method includes all the predictive variables from the loan applicant, while the output is fundamentally the decision to accept or to reject the credit application based on the default risk of the person. Additionally, the mapping from the inputs to the output allows one to transform the internal set of rules of PLTR into transparent feedback about the weaknesses and strengths of the application.

To provide more insights into interpretability, we compare our PLTR model and the random forest in the same Monte Carlo setup as in Section 2, with $p$ fixed to 20, using simple metrics. We consider the two metrics previously defined, i.e., the size of the set of decision rules and the size of a given rule in the decision set. Across the 100 simulations, the random forest registers an average number of 160.9 trees, each with an average number of 410.5 terminal nodes. This leads to a decision set of $410.5 \times 160.9$ binary decision variables or rules that can be used for prediction with this method. Across the same simulations, the average number of active binary decision variables in our penalised logistic regression is equal to $146.9 .^{16}$ Moreover, the number of predicates involved in each of these binary decision variables for our PLTR method varies between 1 and 2 by construction, whereas the maximum number of predicates in a rule of the random forest model is 14.5 on average. Hence, the PLTR model appears to be easier to interpret than the random forest model and comparable to non-linear logistic regression in this sense. ${ }^{17}$

\footnotetext{
${ }^{16}$ Note that for $p=20$ predictors, the maximum number of binary variables is equal to $20+\frac{20 \times 19}{2}=210$. This result illustrates the selection processed through adaptive lasso regression.

${ }^{17}$ The major difference between these two methods is the endogenous character of the thresholds that
} 


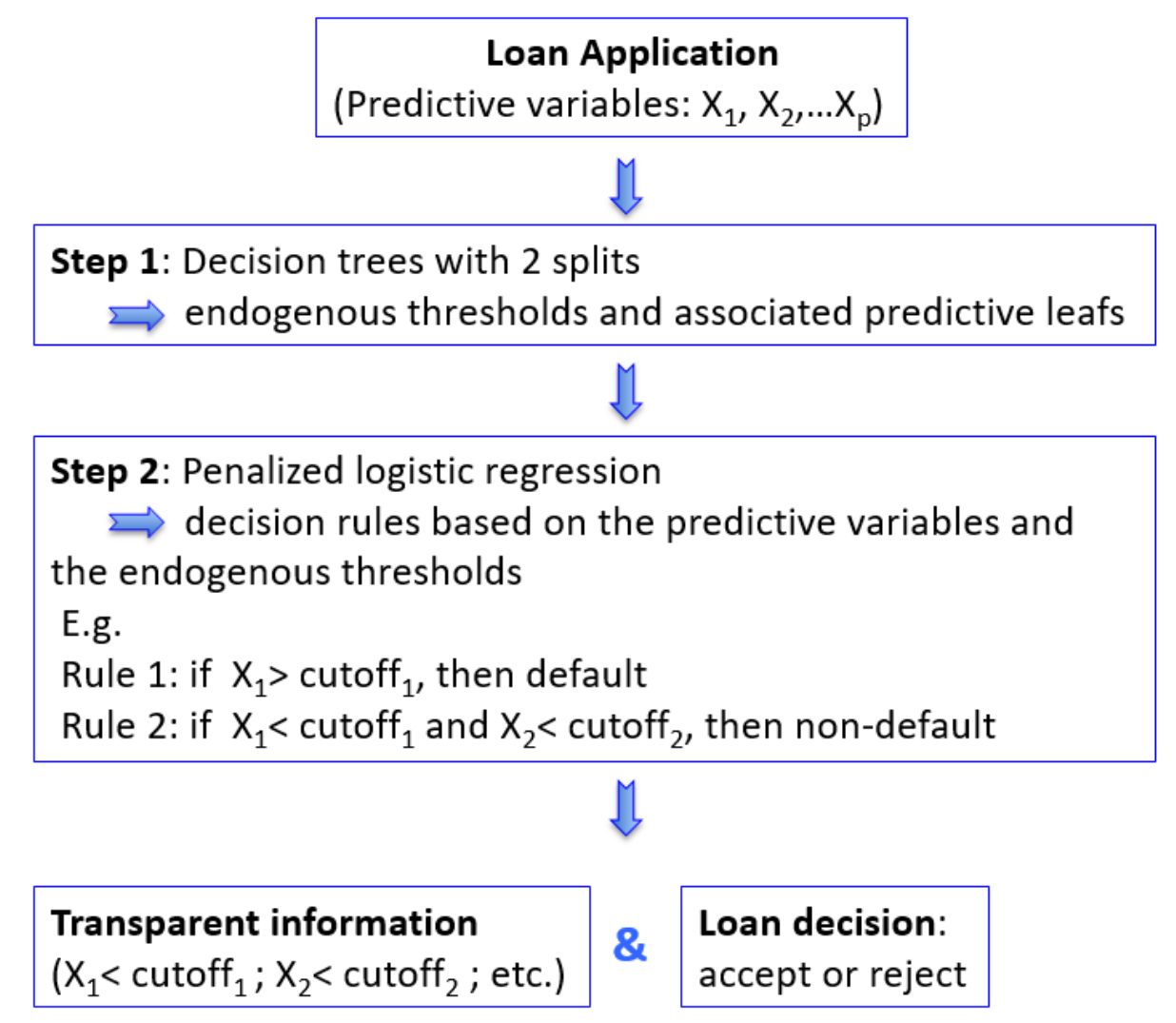

Figure 6: PLTR inference process

Furthermore, marginal effects and elasticities can be easily obtained in PLTR due to the linearity of the link function (cf. Equation 4). On the one hand, this greatly simplifies significance testing as well as the implementation of out-of-sample exercises. On the other hand, this allows credit institutions to easily explain, in a transparent way, the main reasons behind a loan decision.

\section{Model performance with a benchmark dataset}

As a complement to Monte Carlo simulations, we now consider an empirical application based on a benchmark credit default dataset to assess the practical usefulness of PLTR.

\subsection{Data description and processing}

To gauge the out-of-sample performance of the PLTR method and to illustrate its interpretability, we use a popular dataset provided by a financial institution for the Kaggle competition "Give me some credit", which is often used in credit scoring applications (Baesens et al., 2003). The dataset includes several predictive variables and a binary response variable measuring default. The predictive variables provide information about the cuscharacterise variable interactions in our framework. 
tomers (age, monthly income, the number of dependents in the family) and the application form (number of mortgage and real estate loans, the monthly debt payments, the total balance on credit cards, etc.). The dataset contains 10 quantitative predictors. See Table A.1 in Appendix A for a description of the variables in the dataset.

The number of instances in the dataset is equal to 150,000 loans out of which 10,026 defaults, leading to a prior default rate of $0.067 .{ }^{18}$ All the missing values have been replaced by the mean of the predictive variable. Finally, regarding data partitioning, we use the socalled $N \times 2$-fold cross-validation of Dietterich (1998), which involves randomly dividing the dataset into two sub-samples of equal size. The first (second) part is used to build the model, while the second (first) part is used for evaluation. This procedure is repeated $N$ times, and the evaluation metrics are averaged. This method of evaluation produces more robust results compared to classical single data partitioning. We set $N=5$ for computational reasons.

\subsection{Statistical measures of performance and interpretability}

To evaluate the performance of each classifier, we use five accuracy measures considered by Lessmann et al. (2015) in their benchmarking study: the area under the ROC curve (AUC), the Brier score (BS), the Kolmogorov-Smirnov statistic (KS), the percentage of correctly classified (PCC) cases, and the partial Gini index (PGI). These indicators are related to different facets of the predictive performance of scorecards, namely, the accuracy of the scores as measured by the BS statistics, the quality of the classification given by the PCC and KS statistics, and the discriminatory power assessed through the AUC and the PGI statistics. By using several statistics instead of a single one, we expect to obtain a robust and complete evaluation of the relative performances of the competing models.

The AUC tool evaluates the overall discriminatory performance of each model or classifier. It is a measure of the link between the false positive rate (FPR) and the true positive rate (TPR), each computed for every threshold between 0 and 1 . The FPR (TPR) is the percentage of non-defaulted (defaulted) loans misclassified as defaulted (non-defaulted). Thus, AUC reflects the probability that the occurrence of a randomly chosen bad loan is higher than the occurrence of a randomly chosen good loan.

The Gini index is equal to twice the area between the ROC curve and the diagonal. Hence, similar to the AUC metric, it evaluates the discriminatory power of a classifier across several thresholds, with values close to one corresponding to perfect classifications. However, in credit scoring applications, it is not realistic to study all possible thresholds.

\footnotetext{
${ }^{18}$ It is well known that imbalanced classes impede classification: some classifiers may focus too much on the majority class and neglect the minority group (of interest). They can hence exhibit good overall performance despite poorly identifying the minority group, i.e., the borrowers that default. A common solution consists of using an under-sampling or over-sampling method, such as SMOTE. Nonetheless, here, we choose not to resample the data, as the prior default rate is larger than $6 \%$.
} 
Informative thresholds are those located in the lower tail of the distribution of default probabilities (Hand, 2005). Indeed, only applications below a threshold in the lower tail can be granted a credit, which excludes high thresholds. The partial Gini index solves this issue by focusing on thresholds in the lower tail (Pundir and Seshadri, 2012). With $x$ denoting a given threshold and $L(x)$ denoting the function describing the ROC curve, the PGI is then defined as ${ }^{19}$

$$
P G I=\frac{2 \int_{a}^{b} L(x) d x}{(a+b)(b-a)}-1 .
$$

The PCC is the proportion of loans that are correctly classified by the model. Its computation requires discretisation of the continuous variable of estimated probabilities of default. Formally, we need to choose a threshold $\pi$ above (below) which a loan is classified as bad (good). In practise, the threshold $\pi$ is fixed by comparing the costs of rejecting good customers/granting credits to bad customers. Since we do not have such information, we set this threshold to a value such that the predicted number of defaults in the learning sample is equal to the observed number of defaults.

The Kolmogorov-Smirnov statistic is defined as the maximum distance between the estimated cumulative distribution functions of two random variables. In credit scoring applications, these two random variables measure the scores of good loans and bad loans (Thomas et al., 2002).

Lastly, the Brier score (Brier, 1950) is defined as

$$
B S=\frac{1}{n} \sum_{i=1}^{n}\left(\widehat{\operatorname{Pr}}\left(y_{i}=1 \mid x_{i}\right)-y_{i}\right)^{2}
$$

where $\widehat{\operatorname{Pr}}\left(y_{i}=1 \mid x_{i}\right)$ is the estimated probability of default and $y_{i}$ is the target binary default variable. Note that it is the equivalent of the mean-square error but designed for the case of discrete-choice models. Overall, the higher these indicators are, the better the model is, except for the Brier score, for which a smaller value is better.

Regarding the interpretability of the scoring models, the criteria retained to compare PLTR and the random forest method are the size of the decision set and the average size of rules in a decision set (see also Subsection 3.2).

\subsection{Statistical evaluation results}

Table 1 presents the average value of each statistic across the $5 \times 2$ cross-validation test samples. We compare the performance of PLTR to that of traditional logistic regression and the random forest method. Three different versions of the logistic regression are implemented: simple linear logistic regression, its non-linear version, which includes as additional variables

\footnotetext{
${ }^{19}$ The PGI within bounds $a=0$ and $b=1$ is equivalent to the Gini Index. In the empirical applications, we evaluate the PGI within the $(0,0.4)$ bounds as in Lessmann et al. (2015).
} 
quadratic and interaction terms, ${ }^{20}$, and a penalised version of this last model to avoid overfitting due to the large number of predictors. We use the adaptive lasso penalty as described above. These augmented logistic regression models are used to assess the importance of non-linear effects of the features. We also include an SVM and NN in the comparison, as they are widely used for credit scoring applications in the literature (Thomas, 2000; Baesens et al., 2003; Lessmann et al., 2015).

Table 1: Statistical performance indicators: Kaggle dataset

\begin{tabular}{lccccc}
\hline Methods & AUC & PGI & PCC & KS & BS \\
\hline Linear Logistic Regression & 0.6983 & 0.3964 & 0.9082 & 0.3168 & 0.0576 \\
Non-Linear Logistic Regression & 0.7660 & 0.5255 & 0.9127 & 0.4173 & 0.0649 \\
Non-Linear Logistic Regression + ALasso & 0.8062 & 0.6102 & 0.9208 & 0.4751 & 0.0535 \\
Random Forest & 0.8529 & 0.6990 & 0.9260 & 0.5563 & 0.0500 \\
PLTR & 0.8568 & 0.7076 & 0.9247 & 0.5647 & 0.0496 \\
Support Vector Machine & 0.7418 & 0.4830 & 0.9117 & 0.3723 & 0.0619 \\
Neural Network & 0.7517 & 0.5006 & 0.9074 & 0.3895 & 0.0552 \\
\hline
\end{tabular}

Note: Non-linear logistic regression includes linear, quadratic and interaction terms. The method labelled "Non-Linear Logistic Regression + ALasso" corresponds to a penalised version of non-linear logistic regression with an adaptive lasso penalty.

The results displayed in Table 1 show that the random forest method performs better than the three versions of the logistic regression, and this holds for all statistical measures considered. In particular, the differences are more pronounced for the AUC, PGI and KS statistics. Our PLTR method also performs better than the three versions of logistic regression irrespective of the performance measure. This is particularly applicable for the AUC, PGI and KS metrics, for which the dominance is stronger. The take-away message here is that combining decision trees with a standard model such as logistic regression provides a valuable statistical modelling solution for credit scoring. In other words, the non-linearity captured by univariate and bivariate threshold effects obtained from short-depth decision trees can improve the out-of-sample performance of traditional logistic regression. The SVM and NN results are consistent with those in the literature (Thomas, 2000; Baesens et al., 2003; Lessmann et al., 2015; Grennepois et al., 2018). They are slightly better than those of the logistic regression model, but these methods generally perform less well than ensemble learning methods such as the random forest method. Most importantly, these models also perform less well than PLTR.

The results in Table 1 also show that PLTR compares competitively to the random forest method. All statistical performance measures are of the same order. Therefore, the two methods exhibit similar statistical performance, and neither of them should be preferred over the other based on these criteria. However, the parsimony of PLTR contrasts with

\footnotetext{
${ }^{20}$ As already stressed, this non-linear model is the one that is generally used to capture non-linear effects in the framework of logistic regression.
} 
the complexity underlying the prediction rule of the random forest method. To illustrate this point, Table 2 displays the interpretability measures for the random forest method and PLTR, as well as that of linear logistic regression for comparison purposes. The average number of trees in the random forest method across the $5 \times 2$ cross-validation test samples is equal to 173.9. These trees have on average 5,571.1 terminal nodes, with a total of $5,571.1 \times 173.9$ binary variables for prediction (via the majority vote). By contrast, the average number of bivariate threshold effects selected by our penalised logistic regression is only 40. More importantly, these bivariate threshold effects are easily interpretable because they arise from short-depth decision trees. In addition, the PLTR rules are built from only 2 predicates at most, whereas the rules from the random forest method are built from an average number of 32.2 predicates at most. Overall, both criteria confirm that PLTR is easier to interpret than the random forest method. These differences in terms of the size of the decision set and size of the rules between both models are the penalty of capturing more non-linear effects, although such effects do not seem to play a significant role in this dataset. For comparison, the average number of predictors is 11 for linear logistic regression, each of them relying on a single predicate. The PLTR results are not very different from those of linear logistic regression, with the gap corresponding to the non-linear effects included in our model to improve the performance of the benchmark linear logistic regression method.

Table 2: Measures of interpretability: Kaggle dataset

\begin{tabular}{lcc}
\hline Methods & Size of the decision set & Maximal number of predicates \\
\hline Linear Logistic Regression & 11 & 1 \\
Random Forest & $5,571.1 \times 173.9$ & 32.2 \\
PLTR & 40 & 2 \\
\hline
\end{tabular}

Note: This table displays the average values of interpretability measures for linear logistic regression, the random forest method and PLTR.

Lastly, to highlight the interpretability advantage of our method, we report in Table 3 the 10 most important decision rules from short-depth decision trees, which are selected by an adaptive lasso in the implementation of our PLTR method. These decision rules are associated with the largest absolute values of the marginal effects (averaged across individuals). A positive (negative) value of a given marginal effect provides information about the strength of an increase (decrease) of the probability of default. We observe that three univariate threshold variables are selected, i.e., "NumberOfTime60-89DaysPastDueNotWorse $<0.5$ ", "NumberOfTimes90DaysLate $<0.5$ " and

"RevolvingUtilizationOfUnsecuredLines $<0.69814$ ", the first one appearing as the most important in terms of marginal effect. Referring to the description of this variable in Table A.1, we can infer that the probability of default is $3.92 \%$ less important when the number of times a borrower has been between 60 and 89 days past due (but not worse in the last 2 years) is lower than 0.5 compared to the reference case when this number is higher than 0.5. 
Moreover, seven bivariate threshold effects are selected by the models as being important in explaining credit default. This kind of analysis that helps measure through marginal effects the importance of the decision rules from the short-depth decision trees is an important added value of our PLTR model in terms of interpretability.

\section{Robustness across datasets}

In this section, we evaluate the robustness of the above empirical results across datasets. To this end, we consider three popular additional datasets. The first one, named "Housing", is available in an SAS library and has been used by many authors for illustrative examples (Matignon, 2007). The second dataset, labelled the "Australian dataset", concerns credit card applications and is a University of California at Irvine (UCI) dataset provided by Quinlan, and it was used as a credit approval database in the Statlog project. ${ }^{21}$ Lastly, the third dataset, labelled the "Taiwan dataset", is also a UCI dataset that collects information about default payments in Taiwan.

The Housing dataset includes 5, 960 loans, 1, 189 of which defaulted. Therefore, the prior default rate is $19.95 \%$. In the Australian (Taiwan) dataset, there are 690 (30,000) instances out of which $307(6,636)$ defaulted, leading to a prior default rate of $44.49 \%(22.12 \%)$. In the Housing dataset, there are 12 explanatory variables, two of which are nominal. The Australian dataset includes 6 numerical and 8 nominal predictors. For the Taiwan dataset, there are 23 predictors, nine of which are nominal. Tables A.2 and A.3 display the list of predictive variables for the Housing and Taiwan datasets, respectively. We do not provide this information for the Australian dataset, as all attribute names and values have been changed to meaningless symbols to maintain the confidentiality of the data.

We rely on the same $(N \times 2)$ comparison setup as for the benchmark Kaggle dataset, with $N=5$. Table 4 displays the values of the five statistics retained for the comparison of the alternative models. For the Australian dataset, the two best performing models are PLTR and the random forest method, with similar values for all five statistics. ${ }^{22}$ This finding once again confirms the relevance of our approach in terms of statistical performance. The same picture is observed for the Taiwan dataset with the PLTR model appearing as efficient as the random forest method.

Lastly, for the Housing dataset, the random forest method and PLTR are once again the best performing models. However, in contrast to the results obtained for the other

\footnotetext{
${ }^{21}$ StatLog is an international project that aims to compare the performances of machine learning, statistical, and NN algorithms on datasets from real-world industrial areas, including medicine, finance, image analysis, and engineering design.

${ }^{22}$ For the non-linear logistic regression results, we find that all fitted probabilities are higher than 0.6. Therefore, as we compute the PGI within $(0,0.4)$, this statistic cannot be computed. Unlike in practise, this bad performance can also be observed through the high value of the BS statistic compared to those of the other methods.
} 


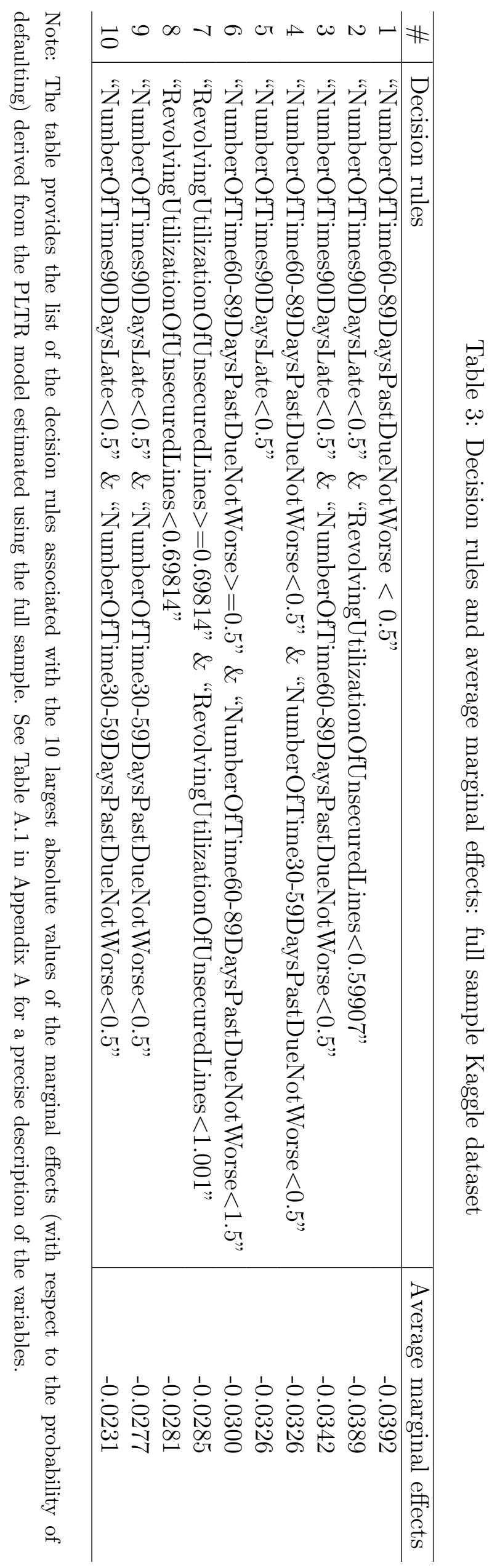


datasets, the random forest model outperforms our method. Table 5 displays the interpretability performance for these three additional datasets. Using the same arguments as above, the average number of active variables (univariate and bivariate threshold effects) in our penalised logistic regression is equal to 47.6, while the random forest method relies on an average of $343.8 \times 110.5$ binary variables for prediction. ${ }^{23}$ Moreover, the results of PLTR are close to those of linear logistic regression for both criteria, indicating that the PLTR model remains interpretable despite including non-linear effects.

Other results, available upon request, show that by relaxing the constraint of parsimony via the inclusion of trivariate and quadrivariate threshold effects, the performance of our penalised logistic regression increases and reaches that of the random forest model. This suggests that complex non-linear relationships that go beyond univariate and bivariate threshold effects are present in this dataset. In view of this result, it is important to emphasise that our method offers a highly flexible framework to credit risk managers, as they can tune their model according to the desired level of parsimony. The predictive performance can be significantly improved but at the cost of less interpretable results.

\section{Economic evaluation}

An important question for a credit risk manager is to what extent these statistical performance gains have a positive impact at a financial level for a credit company. An economic evaluation method consists of estimating the amount of regulatory capital induced by the estimated probabilities of default. A similar comparison approach was proposed by Hurlin et al. (2018) for loss-given-default (LGD) models. However, this approach requires computing other Basel risk parameters, in particular the LGD and the exposure at default (EAD), and hence needs specific information about the consumers and the terms of the loans, which is not publicly available.

An alternative approach consists of comparing the misclassification costs (see Viaene and Dedene, 2004). This cost is estimated from Type 1 and Type 2 errors weighted by their probability of occurrence. Formally, let $C_{F N}$ be the cost associated with a Type 1 error (the cost of granting credit to a bad customer) and $C_{F P}$ be the cost associated with a Type 2 error (e.g., the cost of rejecting a good customer). Thus, the misclassification error cost is defined as

$$
M C=C_{F P} F P R+C_{F N} F N R,
$$

where FPR is the false positive rate and FNR is the false negative rate. There is no consensus in the literature about how to determine $C_{F N}$ and $C_{F P}$. Two alternatives have

\footnotetext{
${ }^{23}$ In this dataset, we identify on average of 110.5 trees in the forest, with an average number of terminal nodes equal to 343.8 for each tree. Furthermore, at most, 18.8 predicates are used on average in the rules of the random forest method against 2 at most for the PLTR model. Hence, PLTR is once again better from the interpretability point of view.
} 
Table 4: Statistical performance indicators: robustness check

\begin{tabular}{|c|c|c|c|c|c|}
\hline Methods & AUC & PGI & $\mathrm{PCC}$ & KS & $\mathrm{BS}$ \\
\hline \multicolumn{6}{|c|}{ Australian dataset } \\
\hline Linear Logistic Regression & 0.8998 & 0.5664 & 0.8374 & 0.7135 & 0.1186 \\
\hline Non-Linear Logistic Regression & 0.6090 & & 0.6067 & 0.2266 & 0.3921 \\
\hline Non-Linear Logistic Regression + ALasso & 0.8866 & 0.5092 & 0.8214 & 0.6816 & 0.1333 \\
\hline Random Forest & 0.9344 & 0.6246 & 0.8603 & 0.7523 & 0.0999 \\
\hline PLTR & 0.9299 & 0.6370 & 0.8606 & 0.7425 & 0.1029 \\
\hline Support Vecto & 0.9210 & 0.5557 & .8445 & 0.7391 & 0.1122 \\
\hline Neur & 0.9141 & 0.5799 & 0.8539 & 0.7366 & 0.1102 \\
\hline \multicolumn{6}{|c|}{ Taiwan dataset } \\
\hline Linear Logistic Regression & 0.6310 & 0.2099 & 0.7586 & 0.2506 & 0.2344 \\
\hline Non-Linear Logistic Re & 0.5963 & 0.0984 & 0.7035 & 0.1927 & 0.2965 \\
\hline Non-Linear Logistic Regression + ALasso & 0.7596 & 0.5029 & 0.7871 & 0.3926 & 0.1447 \\
\hline Random Forest & 0.7722 & 0.4924 & 0.8102 & 0.4177 & 0.1362 \\
\hline PLTR & 0.7780 & 0.5156 & 0.7959 & 0.4257 & 0.1352 \\
\hline upport Vec & 0.7102 & 0.3207 & 0.8195 & 0.3382 & 0.1461 \\
\hline Neural Network & 0.7304 & 0.4226 & 0.7879 & 0.3885 & 0.1401 \\
\hline \multicolumn{6}{|c|}{ Housing dataset } \\
\hline Linear Log & 0.7904 & 0.5508 & 0.8103 & 0.4450 & 0.1228 \\
\hline Non-Linear Logisti & 0.7965 & 0.5425 & 0.8239 & 0.4650 & 0.1199 \\
\hline Non-Linear Logistic Regression + ALasso & 0.8113 & 0.5754 & 0.8217 & 0.4815 & 0.1125 \\
\hline Random Forest & 0.9387 & 0.8157 & 0.9036 & 0.7455 & 0.0736 \\
\hline & 0.9011 & 0.7341 & & 0.6694 & 0.0844 \\
\hline Support Vector 1 & 0.7890 & 0.5514 & 0.8093 & 0.4444 & 0.1254 \\
\hline Neural Network & 0.7910 & 0.5478 & 0.8132 & 0.4470 & 0.1208 \\
\hline
\end{tabular}

Note: Non-linear logistic regression includes linear, quadratic and interaction terms. The method labelled "Non-Linear Logistic Regression + ALasso" corresponds to a penalised version of non-linear logistic regression with the adaptive lasso penalty.

Table 5: Measures of interpretability: robustness check

\begin{tabular}{lcc}
\hline Methods & Size of the decision set & Maximal number of predicates \\
\hline & Australian dataset & \\
\hline Linear Logistic Regression & 34.4 & 1 \\
Random Forest & $52.4 \times 69.6$ & 8 \\
PLTR & 25.4 & 2 \\
\hline \multicolumn{2}{c}{ Taiwan dataset } & 1 \\
\hline Linear Logistic Regression & 78.7 & 29.9 \\
Random Forest & $2,378.7 \times 174.7$ & 2 \\
PLTR & 79.9 & 1 \\
\hline \multicolumn{2}{c}{ Housing dataset } \\
Random Forest & 17 & 18.8 \\
PLTR & $343.8 \times 110.5$ & 2 \\
\hline
\end{tabular}

Note: This table displays the average values of interpretability measures for linear logistic regression, the random forest method and PLTR. 
been proposed. The first method fixes these costs by calibration based on previous studies (Akkoc, 2012). For example, West (2000) set $C_{F N}$ to 5 and $C_{F P}$ to 1 . The second method evaluates misclassification costs for different values of $C_{F N}$ to test as many scenarios as possible (Lessmann et al., 2015). Although there is no consensus on how to determine these costs, it is generally acknowledged that the cost of granting credit to a bad customer is higher than the opportunity cost of rejecting a good customer (see Thomas et al., 2002; West, 2000; Baesens et al., 2003, among others). We choose to follow the second approach to assess the performance of the competing models. We fix $C_{F P}$ at 1 without loss of generality (Hernández-Orallo et al., 2011) and consider values of $C_{F N}$ between 2 and 50. Once these misclassification costs are computed, we set the linear logistic regression as the reference, and we compute the financial gain or cost reduction associated with an alternative scoring model relative to this reference. ${ }^{24}$

Figures A.1-A.4 in Appendix A display the cost reduction or financial gains for the four datasets considered above. First, all methods deliver positive cost reductions, except in three cases. This means that financial institutions relying on each of these methods rather than on the benchmark linear logistic regression are expected to save an amount equivalent to the cost of rejecting (accepting) good (bad) applicants. In view of the large number of credits in bank credit portfolios, these gains could represent substantial savings for credit institutions. The fact that non-linear logistic regression leads to an increase in costs compared to the linear logistic regression comes from the relatively high number of variables in the two datasets (14 and 23 in the Australian and Taiwan datasets, respectively). This leads to a proliferation of predictors (squares of the variables, cross-products of the variables) and therefore to overfitting. The penalised version of the non-linear logistic regression succeeds in dealing with this issue, which materialises in positive values of cost reductions in all cases except for the Australian dataset. The NN and SVM both reduce the misclassification costs compared to the logistic regression. This result is once again consistent with the results of the literature.

Second, across all datasets, the PLTR method is among the most efficient in terms of cost reduction. For the Kaggle dataset, the cost reduction relative to the linear logistic regression is equal to $18.06 \%$ on average. This result also holds in the Taiwan dataset, with an average cost reduction of $22.29 \%$. Note that the random forest method leads to lower cost reduction for these two datasets, with an average cost reduction of $13.09 \%$ (11.51\%) for the Kaggle (Taiwan) dataset. This means that although the random forest method has high global predictive accuracy, as given by the proportion of correct classification (see Tables 1 and 4), it fails to some extent to detect bad customers, which leads to a relative increase in costs due to more false negatives. For the other two datasets (Australian and Housing), the random forest method performs well. With the Australian dataset, the average cost reduction of the

\footnotetext{
${ }^{24}$ The misclassification costs are computed from test samples.
} 
random forest (PLTR) method is equal to $22.71 \%$ (14.89\%). For the Housing dataset, the average values are equal to $44.56 \%$ and $38.69 \%$ for the random forest method and PLTR, respectively.

We also consider a second measure of performance, namely, the expected maximum profit (EMP) introduced by Verbraken et al. (2014), to compare the models from an economic viewpoint. The EMP takes into account the profits received by the non-defaulters and the losses caused by the defaulters. This allows us to compute an EMP value that is expressed as a percentage of the total loan amount and measures the incremental profit relative to not building a credit scoring model. The EMP is based on the following utility function of the decision maker:

$$
P\left(t ; b, c, c^{*}\right)=\left(b-c^{*}\right) \pi_{0} F_{0}(t)-\left(c+c^{*}\right) \pi_{1} F_{1}(t)
$$

where $t$ is a cutoff; $b$ is the benefit associated with a true positive; $c$ is the cost associated with a false positive; $c^{*}$ is the cost associated with an individual case; $\pi_{0}$ and $\pi_{1}$ are the prior probabilities of non-default and default, respectively; and $F_{0}(t)$ and $F_{1}(t)$ are the corresponding cumulative density functions. The parameters $b$ and $c$ are calibrated using the LGD and return on investment (ROI, see Verbraken et al., 2014 for more details). ${ }^{25}$ Since our datasets do not include information on the LGD or the ROI, to calculate the EMP, we assume that the LGD distribution is bimodal, with a probability of complete recovery equal to 0.55 and a probability of complete loss of 0.1 , and that the ROI per granted loan is fixed to $26.44 \%$ for all the credits, which corresponds to the value considered by Verbraken et al. (2014) for their illustrations.

Tables 6 and 7 report the results obtained for the Kaggle dataset and for the three datasets considered in the robustness analysis. For the Kaggle dataset, the EMP analysis confirms that the PLTR method generates more profit $(0.4387 \%)$ than the different versions of logistic regression (from $0.1910 \%$ to $0.3730 \%$ ). Furthermore, PLTR exhibits similar economic performance to random forest $(0.4169 \%)$, while keeping the intrinsic interpretability of logistic regression. Similar qualitative results are obtained for the three other datasets of our robustness analysis. These results confirm those previously obtained with the misclassification cost analysis (Viaene and Dedene, 2004).

To conclude, all results show that the PLTR model may generate important cost reductions compared to the standard logistic regression model generally used by the credit risk industry while preserving its intrinsic interpretability.

\section{Conclusion}

Despite the development and dissemination of many efficient machine learning classification algorithms, the benchmark scoring model in the credit industry remains logistic regression.

\footnotetext{
${ }^{25}$ To implement the EMP measure, we use the R package EMP (July 2019).
} 
Table 6: Economic performance indicator: Kaggle dataset

\begin{tabular}{lc}
\hline Methods & Expected Maximum Profit (in \%) \\
\hline Linear Logistic Regression & 0.1910 \\
Non-Linear Logistic Regression & 0.2925 \\
Non-Linear Logistic Regression + ALasso & 0.3730 \\
Random Forest & 0.4169 \\
PLTR & 0.4387 \\
Support Vector Machine & 0.2846 \\
Neural Network & 0.2384 \\
\hline
\end{tabular}

Note: Non-linear logistic regression includes linear, quadratic and interaction terms. The method labelled "Non-Linear Logistic Regression + ALasso" corresponds to a penalised version of non-linear logistic regression with an adaptive lasso penalty.

Table 7: Economic performance indicator: robustness check

\begin{tabular}{|c|c|}
\hline Methods & aximum Profit (in \%) \\
\hline \multicolumn{2}{|c|}{ Australian dataset } \\
\hline Linear Logistic Regression & 10.0124 \\
\hline Non-Linear Logistic Regression & 6.6850 \\
\hline Non-Linear Logistic Regression + ALasso & 9.6215 \\
\hline Random Forest & 10.2572 \\
\hline PLTR & 10.1842 \\
\hline Support Vector Machine & 10.2002 \\
\hline Neural Network & 10.0733 \\
\hline \multicolumn{2}{|c|}{ Taiwan dataset } \\
\hline Linear Logistic Regression & 1.5162 \\
\hline Non-Linear Logistic Regression & 1.2827 \\
\hline Non-Linear Logistic Regression + ALasso & 2.1365 \\
\hline Random Forest & 2.2630 \\
\hline PLTR & 2.3075 \\
\hline Support Vector Machine & 1.8842 \\
\hline Neural Network & 2.1235 \\
\hline \multicolumn{2}{|c|}{ Housing dataset } \\
\hline Linear Logistic Regression & 2.0220 \\
\hline Non-Linear Logistic Regression & 2.2434 \\
\hline Non-Linear Logistic Regression + ALasso & 2.2924 \\
\hline Random Forest & 3.7940 \\
\hline PLTR & 3.3660 \\
\hline Support Vector Machine & 2.0189 \\
\hline Neural Network & 2.0765 \\
\hline
\end{tabular}

Note: Non-linear logistic regression includes linear, quadratic and interaction terms. The method labelled "Non-Linear Logistic Regression + ALasso" corresponds to a penalised version of non-linear logistic regression with an adaptive lasso penalty. 
This current state is caused mainly by the stability and robustness of the logistic regression model and also its intrinsic interpretability. Many academic papers advocate the use of more sophisticated ensemble methods, such as the random forest method. These blackbox models are not interpretable, but many agnostic methods can be used to make their forecasting rules interpretable ex post for the various stakeholders (risk modellers, model checkers, clients, management, regulators, etc.). Nevertheless, these alternative models are still generally considered challenger models and rarely used in the credit granting process or for regulatory purposes.

Recognising that traditional logistic regression underperforms random forest due to its pitfalls in modelling non-linear (threshold and interaction) effects, this article introduces penalised logistic tree regression (PLTR) with predictive variables given by easy-to-interpret endogenous univariate and bivariate threshold effects. These effects are quantified by dummy variables associated with leaf nodes of short-depth decision trees built with couples of the original predictive variables. Our main objective is to combine decision trees (from the field of machine learning) and a logistic regression model (from the field of econometrics) to obtain the best of both worlds: a performing and interpretable hybrid credit scoring model.

Monte Carlo simulations and an empirical application based on four real-life credit scoring datasets show that PLTR has good predictive power while remaining easily interpretable. More precisely, using several metrics to evaluate both the accuracy and the interpretability of credit models, we show that it performs better than traditional linear and non-linear logistic regression, while being competitive relative to the random forest method. We also evaluate the economic benefit of using our PLTR method through misclassification costs and expected maximum profit analysis. We find that beyond parsimony, the PLTR method leads to a significant reduction in misclassification costs. 


\section{A Appendix A: Additional Figures and Tables}

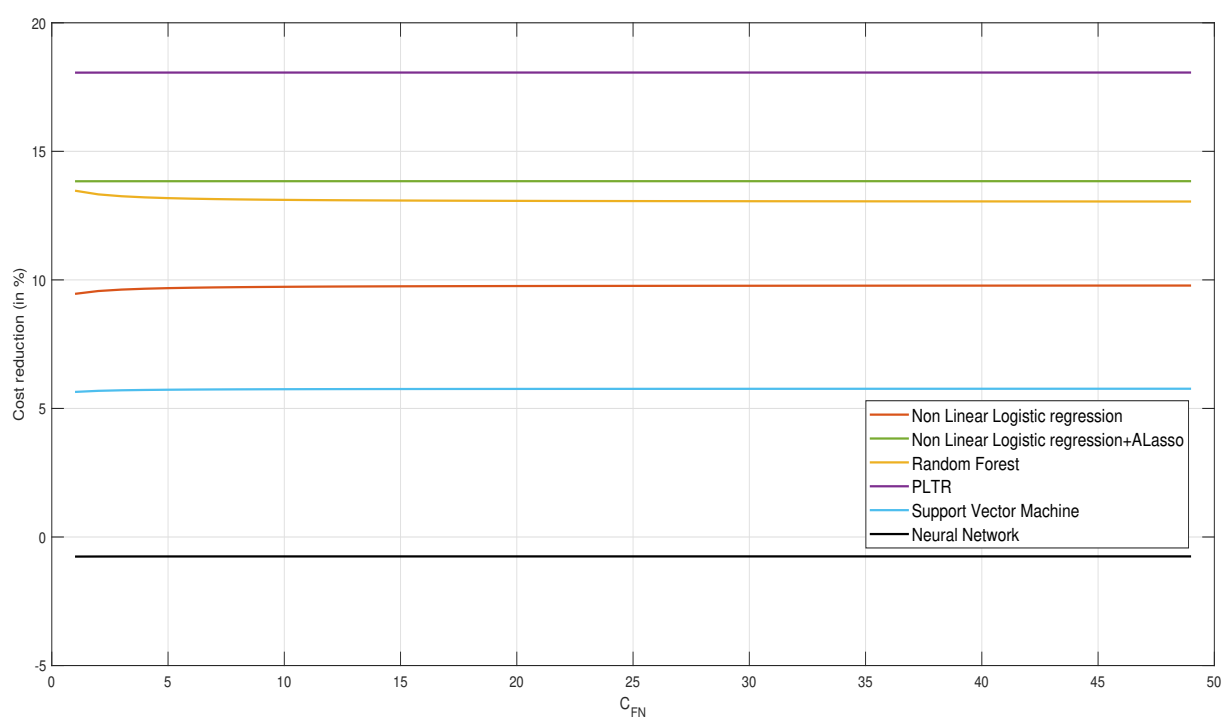

Figure A.1: Economic evaluation for the Kaggle dataset

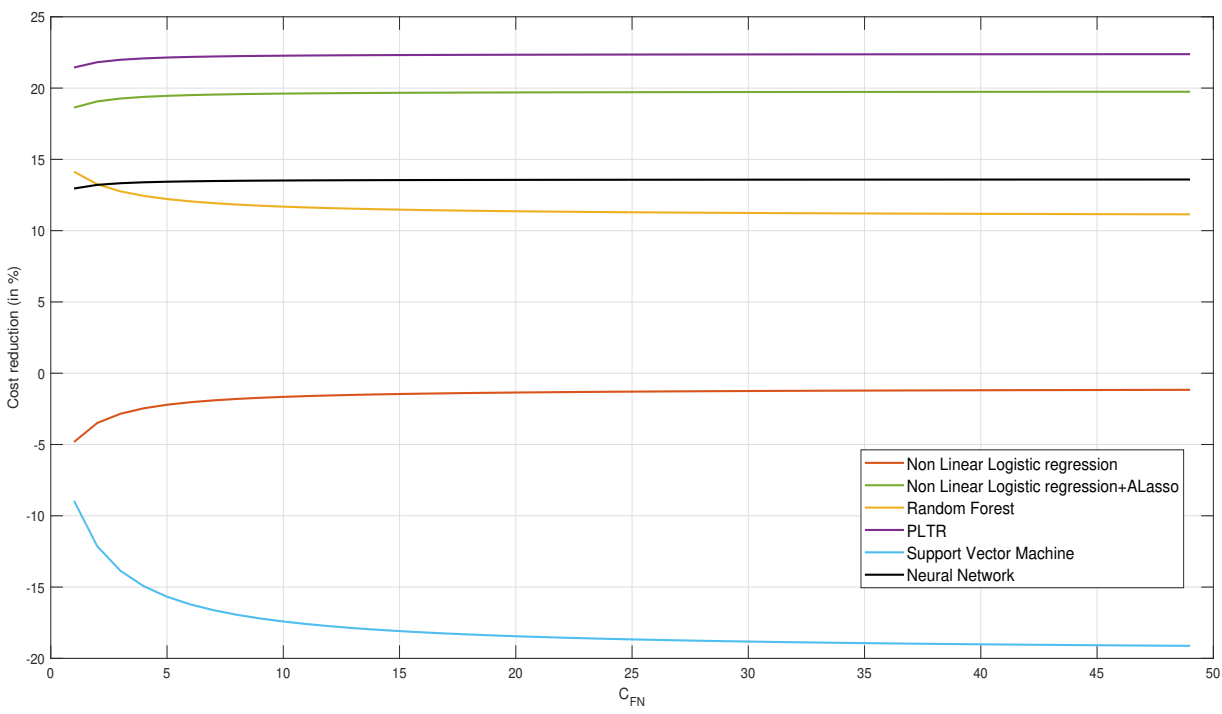

Figure A.2: Economic evaluation for the Taiwan dataset 


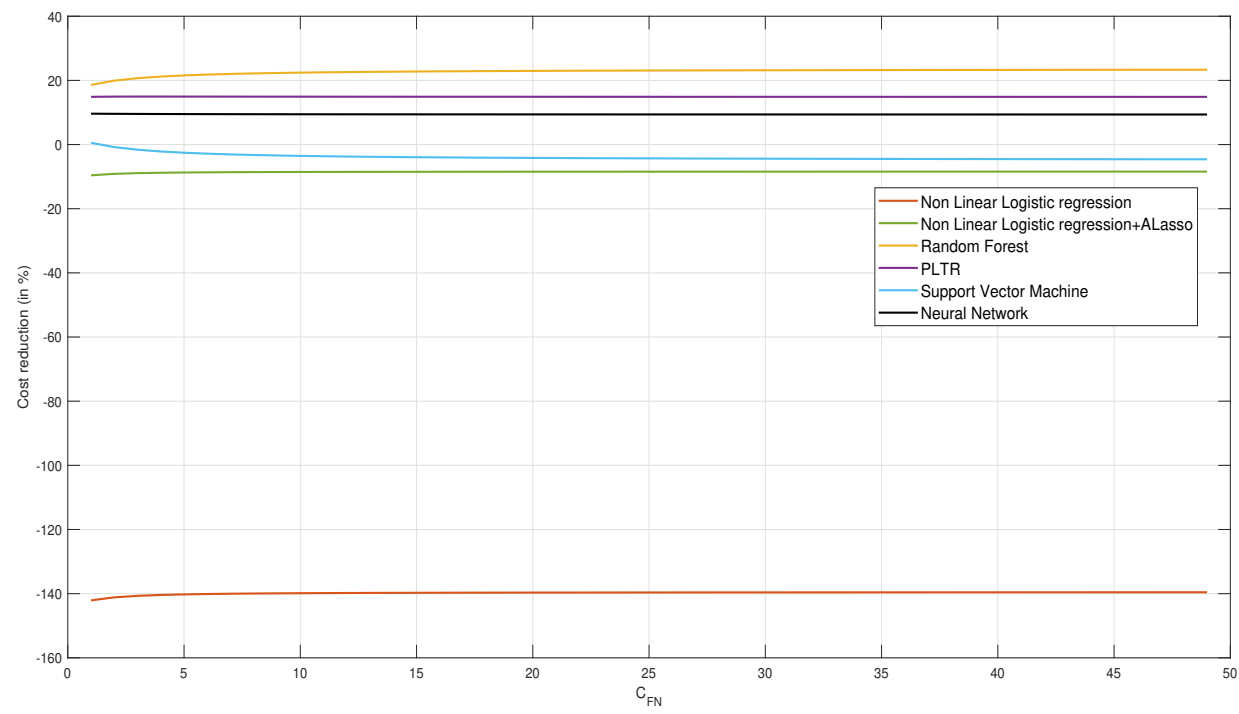

Figure A.3: Economic evaluation for the Australian dataset

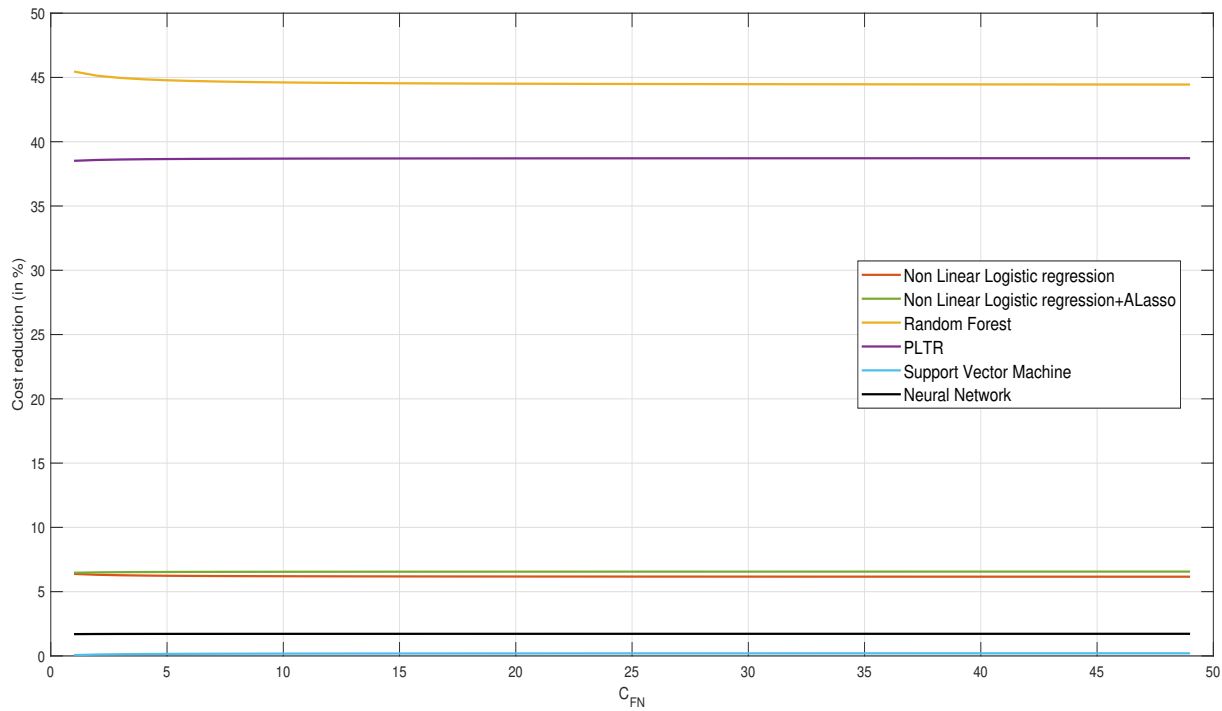

Figure A.4: Economic evaluation for the Housing dataset 
Table A.1: Description of the variables in the Kaggle dataset "Give me some credit"

\begin{tabular}{|c|c|c|}
\hline Variable & Type & Description \\
\hline SeriousDlqin2yrs & Binary & $\begin{array}{l}\text { The person experienced } 90 \text { days } \\
\text { past due delinquency or worse } \\
\text { (Yes/No) }\end{array}$ \\
\hline RevolvingUtilizationOfUnsecuredLines & Percentage & $\begin{array}{l}\text { Total balance on credit cards and } \\
\text { personal lines of credit except } \\
\text { real estate and no instalment } \\
\text { debt such as car loans divided by } \\
\text { the sum of credit limits }\end{array}$ \\
\hline Age & Interval & Age of the borrower (in years) \\
\hline NumberOfTime30-59DaysPastDueNotWorse & Interval & $\begin{array}{l}\text { Number of times a borrower has } \\
\text { been between } 30 \text { and } 59 \text { days } \\
\text { past due but not worse in the } \\
\text { last } 2 \text { years }\end{array}$ \\
\hline DebtRatio & Percentage & $\begin{array}{l}\text { Monthly debt payments, alimony } \\
\text { and living costs over the monthly } \\
\text { gross income }\end{array}$ \\
\hline MonthlyIncome & Interval & Monthly Income \\
\hline NumberOfOpenCreditLinesAndLoans & Interval & $\begin{array}{l}\text { Number of open loans (like car } \\
\text { loan or mortgage) and credit } \\
\text { lines (credit cards) }\end{array}$ \\
\hline NumberOfTimes90DaysLate & Interval & $\begin{array}{l}\text { Number of times a borrower has } \\
\text { been } 90 \text { days or more past due }\end{array}$ \\
\hline NumberRealEstateLoansOrLines & Interval & $\begin{array}{l}\text { Number of mortgage and real } \\
\text { estate loans including home } \\
\text { equity lines of credit }\end{array}$ \\
\hline NumberOfTimes60-89DaysPastDueNotWorse & Interval & $\begin{array}{l}\text { Number of times a borrower has } \\
\text { been between } 60 \text { and } 89 \text { days } \\
\text { past due but not worse in the } \\
\text { last } 2 \text { years }\end{array}$ \\
\hline NumberOfDependents & Interval & $\begin{array}{l}\text { Number of dependents in family } \\
\text { excluding themselves (spouse, } \\
\text { children, etc.) }\end{array}$ \\
\hline
\end{tabular}


Table A.2: Description of the variables in the Housing dataset

\begin{tabular}{|l|l|l|}
\hline Variable & Type & Description \\
\hline Bad & Binary & Whether the consumer had a default on the loan (1) or not (0) \\
Clage & Interval & Age of the oldest trade (in months) \\
Clno & Interval & Number of trades \\
Debtinc & Interval & Ratio of debt to income \\
Delinq & Interval & Number of neglectful trades \\
Derog & Interval & Number of major derogatory reports \\
Job & Nominal & Professional categories \\
Loan & Interval & Amount of the loan \\
Mortdue & Interval & Amount due on the mortgage \\
Ninq & Interval & Number of recent credits enquired \\
Reason & Binary & Whether the loan is for debt consolidation (DebtCon) or home \\
& & improvement (HomeImp) \\
Value & Interval & Current property value \\
Yoj & Interval & Number of years at the present job \\
\hline
\end{tabular}

Table A.3: Description of the variables in the Taiwan dataset

\begin{tabular}{|c|c|c|}
\hline Variable & Type & Description \\
\hline $\mathrm{Y}$ & Binary & default payment $($ Yes $=1, \mathrm{No}=0)$ \\
\hline $\mathrm{X} 1$ & Quantitative & Amount of the given credit (NT dollar) \\
\hline $\mathrm{X} 2$ & Binary & Gender $(1=$ male; $2=$ female $)$ \\
\hline $\mathrm{X} 3$ & Nominal & $\begin{array}{l}\text { Education }(1=\text { graduate school; } 2=\text { university; } 3=\text { high school; } \\
4=\text { others })\end{array}$ \\
\hline $\mathrm{X} 4$ & Nominal & Marital status $(1=$ married; $2=$ single; $3=$ others $)$ \\
\hline $\mathrm{X} 5$ & Quantitative & Age (year) \\
\hline $\mathrm{X} 6-\mathrm{X} 11$ & Nominal & $\begin{array}{l}\text { X6 - X11: History of past payment. We tracked the past monthly } \\
\text { payment records (from April to September, 2005) as follows: } \\
\text { X6 = the repayment status in September, } 2005 ; \text { X } 7=\text { the } \\
\text { repayment status in August, } 2005 ; . . \text {. X } 11=\text { the repayment } \\
\text { status in April, } 2005 . \text { The measurement scale for the } \\
\text { repayment status is: }-1=\text { pay duly; } 1=\text { payment delay for one } \\
\text { month; } 2=\text { payment delay for two months; } . . ; 8=\text { payment } \\
\text { delay for eight months; } 9=\text { payment delay for nine months and above }\end{array}$ \\
\hline $\mathrm{X} 12-\mathrm{X} 17$ & Quantitative & $\begin{array}{l}\text { Amount of bill statement (NT dollars). X12 = amount of bill state- } \\
\text { ment in September, 2005; X13 = amount of bill statement in August, } \\
2005 ; . \text {. . X17 = amount of bill statement in April, } 2005\end{array}$ \\
\hline $\mathrm{X} 18-\mathrm{X} 23$ & Quantitative & $\begin{array}{l}\text { Amount of previous payment (NT dollars). X18 = amount paid in } \\
\text { September, 2005; X19 = amount paid in August, 2005; . . ; } \\
\text { X23 = amount paid in April, } 2005\end{array}$ \\
\hline
\end{tabular}




\section{Bibliography}

ACPR (2020). Governance of artificial intelligence in finance. Discussion papers publication, November, 2020.

Akkoc, S. (2012). An empirical comparison of conventional techniques, neural networks and the three stage hybrid adaptive neuro fuzzy inference system (ANFIS) model for credit scoring analysis: The case of Turkish credit card data. European Journal of Operational Research, 222(1):168-178.

Altman, E. I. (1968). Financial ratios, discriminant analysis and the prediction of corporate bankruptcy. The Journal of Finance, 23(4):589-609.

Athey, S. (2018). The impact of machine learning on economics. In The economics of artificial intelligence: An agenda, pages 507-547. University of Chicago Press.

Athey, S. and Imbens, G. W. (2019). Machine learning methods that economists should know about. Annual Review of Economics, 11:685-725.

Baesens, B., Gestel, T. V., Viaene, S., Stepanova, M., Suykens, J., and Vanthienen, J. (2003). Benchmarking state-of-the-art classification algorithms for credit scoring. Journal of the Operational Research Society, 54:627-635.

Bauweraerts, J. (2016). Predicting bankruptcy in private firms: Towards a stepwise regression procedure. International Journal of Financial Research, 7(2):147-153.

Bracke, P., Datta, A., Jung, C., and Sen, S. (2019). Machine learning explainability in finance: an application to default risk analysis. Bank of England, Staff Working Paper No. 816 .

Breiman, L. (1996). Bagging predictors. Machine Learning, 26:123-140.

Breiman, L. (2001). Random forest. Machine Learning, 45:5-32.

Brier, G. W. (1950). Verification of forecasts expressed in terms of probability. Monthly weather review, 78(1).

Bussman, N., Giudici, P., Marinelli, D., and Papenbrock, J. (2019). Explainable machine learning in credit risk management. Working paper SSRN, available at http://dx.doi.org/10.2139/ssrn.3506274.

Cardell, N. S. and Steinberg, D. (1998). The hybrid-CART logit model in classification and data mining. Working paper, Salford-System. 
Chan, K. Y. and Loh, W. Y. (2004). Lotus: An algorithm for building accurate and comprehensible logistic regression trees. Journal of Computational and Graphical Statistics, $13(4): 826-852$.

Charpentier, A., Flachaire, E., and Ly, A. (2018). Econometrics and machine learning. Economics and Statistics, 505(1):147-169.

Coffman, J. (1986). The proper role of tree analysis in forecasting the risk behavior of borrowers. Management Decision Systems, Atlanta, MDS Reports, 3(4):7.

De Caigny, A., Coussement, K., and De Bock, K. W. (2018). A new hybrid classification algorithm for customer churn prediction based on logistic regression and decision trees. European Journal of Operational Research, 269(2):760-772.

Deng, H. (2019). Interpreting tree ensemble with intrees. International Journal of Data Science and Analytics, 7(4):277-287.

Desai, V. S., Crook, J. N., and Overstreet Jr, G. A. (1996). A comparison of neural networks and linear scoring models in the credit union environment. European Journal of Operational Research, 95(1):24-37.

Dietterich, T. G. (1998). Approximate statistical tests for comparing supervised classification learning algorithms. Neural Computation, 10(7):1895-1923.

Doshi-Velez, F. and Kim, B. (2017). Towards a rigorous science of interpretable machine learning. arXiv preprint arXiv:1702.08608.

Durand, D. (1941). Risk elements in consumer installment financing. National Bureau of Economic Research, New York.

EBA (2020). Report on big data and advanced analytics. European Banking Authority, January, 2020.

EC (2020). White paper on artificial intelligence: A european approach to excellence and trust. European Commission, February, 2020.

Fan, J. and Li, R. (2001). Variable selection via nonconcave penalized likelihood and its oracle properties. Journal of the American Statistical Association, 96(456):1348-1360.

Finlay, S. (2011). Multiple classifier architectures and their application to credit risk assessment. European Journal of Operational Research, 210(2):368-378.

Friedman, J., Hastie, T., and Tibshirani, R. (2010). Regularization paths for generalized linear models via coordinate descent. Journal of Statistical Software, 33(1):1-22. 
Frost, J., Gambacorta, L., Huang, Y., Shin, H. S., and Zbinden, P. (2019). Bigtech and the changing structure of financial intermediation. Economic Policy.

Grennepois, N., Alvirescu, M., and Bombail, M. (2018). Using random forest for credit risk models. Deloitte Risk Advisory, September, 2018.

Grennepois, N. and Robin, E. (2019). Explain artificial intelligence for credit risk management. Deloitte Risk Advisory, July, 2019.

Hand, D. J. (2005). Good practice in retail credit scorecard assessment. Journal of the Operational Research Society, 56(9):1109-1117.

Henley, W. and Hand, D. (1996). A k-nearest-neighbour classifier for assessing consumer credit risk. The Statistician, 45(1):77-95.

Henley, W. E. and Hand, D. J. (1997). Construction of a k-nearest neighbour credit-scoring system. IMA Journal of Mathematics Applied in Business and Industry, 8:305-321.

Hernández-Orallo, J., Flach, P. A., and Ramirez, C. F. (2011). Brier curves: a new costbased visualisation of classifier performance. In ICML, pages 585-592.

Hoerl, A. E. and Kennard, R. W. (1970). Ridge regression: Biased estimation for nonorthogonal problems. Technometrics, 12(1):55-67.

Hurlin, C., Leymarie, J., and Patin, A. (2018). Loss functions for loss given default model comparison. European Journal of Operational Research, 268(1):348-360.

Hurlin, C. and Pérignon, C. (2019). Machine learning et nouvelles sources de données pour le scoring de crédit. Revue d'économie financière, (3):21-50.

Landwehr, N., Hall, M., and Frank, E. (2005). Logistic model trees. Machine Learning, 59:161-205.

Lessmann, S., Baesens, B., Seow, H.-V., and Thomas, L. C. (2015). Benchmarking stateof-the-art classification algorithms for credit scoring: An update of research. European Journal of Operational Research, 247:124-136.

Lundberg, S. M. and Lee, S.-I. (2017). A unified approach to interpreting model predictions. Advances in Neural Information Processing Systems, pages 4765-4774.

Makowski, P. (1985). Credit scoring branches out. Credit World, 75(1):30-37.

Martens, D., Baesens, B., and Van Gestel, T. (2008). Decompositional rule extraction from support vector machines by active learning. IEEE Transactions on Knowledge and Data Engineering, 21(2):178-191. 
Matignon, R. (2007). Data Mining Using SAS Enterprise Miner.

McIlhagga, W. H. (2016). penalized: A matlab toolbox for fitting generalized linear models with penalties.

Molnar, C. (2019). Interpretable machine learning. A Guide for Making Black Box Models Explainable. https://christophm.github.io/interpretable-ml-book/.

Mullainathan, S. and Spiess, J. (2017). Machine learning: an applied econometric approach. Journal of Economic Perspectives, 31(2):87-106.

Óskarsdóttir, M., Bravo, C., Sarraute, C., Vanthienen, J., and Baesens, B. (2019). The value of big data for credit scoring: Enhancing financial inclusion using mobile phone data and social network analytics. Applied Soft Computing, 74:26-39.

Paleologo, G., Elisseeff, A., and Antonini, G. (2010). Subagging for credit scoring models. European Journal of Operational Research, 201(2):490-499.

Park, M. Y. and Hastie, T. (2007). L1-regularization path algorithm for generalized linear models. Journal of the Royal Statistical Society, 69(4):659-677.

Pundir, S. and Seshadri, R. (2012). A novel concept of partial Lorenz curve and partial Gini index. International Journal of Engineering, Science and Innovative Technology, $1: 296-301$.

Setiono, R., Baesens, B., and Mues, C. (2008). Recursive neural network rule extraction for data with mixed attributes. IEEE Transactions on Neural Networks, 19(2):299-307.

Shewchuk, J. R. et al. (1994). An introduction to the conjugate gradient method without the agonizing pain. Carnegie-Mellon University. Department of Computer Science.

Srinivasan, V. and Kim, Y. H. (1987). Credit granting: A comparative analysis of classification procedures. The Journal of Finance, 42(3):665-681.

Steenackers, M. and Goovaerts, J. (1989). A credit scoring model for personal loans. Insurance: Mathematics and Economics, 8(1):31-34.

Stepanova, M. and Thomas, L. C. (2001). Phab scores: Proportional hazards analysis behavioural scores. The Journal of the Operational Research Society, 52(9):1007-1016.

Tam, K. Y. and Kiang, M. Y. (1992). Managerial applications of neural networks: the case of bank failure predictions. Management Science, 38(7):926-947.

Thomas, L., Crook, J., and Edelman, D. (2017). Credit scoring and its applications. SIAM. 
Thomas, L. C. (2000). A survey of credit and behavioural scoring: forecasting financial risk of lending to consumers. International journal of forecasting, 16(2):149-172.

Thomas, L. C., Edelman, D. B., and Crook, J. N. (2002). Credit scoring and its application.

Tibshirani, R. (1996). Regression shrinkage and selection via the lasso. Journal of the Royal Statistical Society, 58(1):267-288.

Varian, H. R. (2014). Big data: New tricks for econometrics. Journal of Economic Perspectives, 28(2):3-28.

Verbraken, T., Bravo, C., Weber, R., and Baesens, B. (2014). Development and application of consumer credit scoring models using profit-based classification measures. European Journal of Operational Research, 238(2):505-513.

Viaene, S. and Dedene, G. (2004). Cost-sensitive learning and decision making revisited. European Journal of Operational Research, 166:212-220.

Wang, W. (2012). How the small and medium-sized enterprises' owners' credit features affect the enterprises' credit default behavior? E3 Journal of Business Management and Economics, 3(2):90-95.

West, D. (2000). Neural network credit scoring models. Computers $\&$ Operations Research, 27(11-12):1131-1152.

Yobas, M. B., Crook, J. N., and Ross, P. (2000). Credit scoring using neural and evolutionary techniques. IMA Journal of Mathematics Applied in Business and Industry, 11:111-125.

Zhang, H. H. and Lu, W. (2007). Adaptive lasso for Cox's proportional hazards model. Biometrika, 94(3):691-703.

Zou, H. (2006). The adaptive lasso and its oracle properties. Journal of the American Statistical Association, 101(476):1418-1429.

Zou, H. and Hastie, T. (2005). Regularization and variable selection via the elastic net. Journal of the Royal Statistical Society, 67(2):301-320. 